\title{
Semigroup presentations for test local groups
}

\author{
J. C. Costa \\ C. Nogueira \\ M. L. Teixeira
}

March 9, 2014

\begin{abstract}
In this paper we exhibit a type of semigroup presentations which determines a class of local groups. We show that the finite elements of this class generate the pseudovariety $\mathbf{L G}$ of all finite local groups and use them as test-semigroups to prove that $\mathbf{L G}$ and $\mathbf{S}$, the pseudovariety of all finite semigroups, verify the same $\kappa$-identities involving $\kappa$-terms of rank at most 1 , where $\kappa$ denotes the implicit signature consisting of the multiplication and the $(\omega-1)$-power.
\end{abstract}

Keywords. Local group, semigroup presentation, Rees matrix semigroup, pseudovariety, $\kappa$-term, canonical form.

\section{Introduction}

Let $A$ be an alphabet. We denote by $A^{*}$ the free monoid of all finite words in $A$ (including the empty word 1) and by $A^{+}$the free semigroup of all finite non-empty words in $A$. A semigroup presentation is an ordered pair $\langle A \mid R\rangle$, with $R \subseteq A^{+} \times A^{+}$. A semigroup $S$ is said to be defined by the presentation $\langle A \mid R\rangle$ if $S$ is isomorphic to the quotient semigroup $A^{+} / \rho_{R}$, where $\rho_{R}$ is the smallest congruence on $A^{+}$containing $R$. If $(u, v) \in \rho_{R}$ then $u$ and $v$ represent the same element of $S$ and so it is usual to denote $(u, v)$ by $u=v$. However, when we want to avoid ambiguity, we denote $u \equiv v$ to assure that $u$ and $v$ are precisely the same word over $A$. See [10] for an introduction to this topic.

A local group is a semigroup $S$ such that $e S e$ is a group for each idempotent $e$ of $S$. The finite local groups form a pseudovariety (i.e., a class of finite semigroups closed under taking subsemigroups, homomorphic images and finite direct products) usually denoted by LG. More generally, if $\mathbf{H}$ is a pseudovariety of groups then $\mathbf{L H}$ denotes the pseudovariety

J. C. Costa \& M. L. Teixeira: CMAT, Dep. Matemática e Aplicações, Universidade do Minho, Campus de Gualtar, 4700-320 Braga, Portugal; e-mail: jcosta@math.uminho.pt, mlurdes@math.uminho.pt

C. Nogueira: CMAT, Escola Superior de Tecnologia e Gestão, Instituto Politécnico de Leiria, Campus 2, Morro do Lena, Alto Vieiro, 2411-901 Leiria, Portugal; e-mail: conceicao.veloso@ipleiria.pt

Mathematics Subject Classification (2010): 20M05, 20M07 
of all finite semigroups $S$ such that $e S e \in \mathbf{H}$ for each idempotent $e$ of $S$, and we recall that $\mathbf{L H}=\mathbf{H} * \mathbf{D}[13$, where $\mathbf{D}$ is the pseudovariety of all finite semigroups whose idempotents are right zeros. It is well known (see [13] for a proof) that a finite semigroup $S$ is a local group if and only if all the idempotents of $S$ lie in the minimal ideal of $S$. A proof of the generalization of this result to arbitrary semigroups can be found in Proposition 2.1 below. There, a semigroup $S$ is characterized as being a local group if and only if $S$ has no idempotents or $S$ has a minimal ideal $J$ which is a completely simple semigroup that contains all the idempotents of $S$. In this case, by the Rees-Suschkewitsch Theorem, $J$ is isomorphic to a Rees matrix semigroup over a group (the maximal subgroup of $S$ ). In [9], Howie and Ruškuc showed how to find a semigroup presentation for a Rees matrix semigroup $\mathcal{M}[G ; I, \Lambda ; P]$ given a semigroup presentation for the group $G$.

In [2] (see also [1, Section 10.6]), Almeida and Azevedo showed that a semidirect product of the form $\mathbf{V} * \mathbf{D}$, with the pseudovariety $\mathbf{V}$ not locally trivial, is generated by a class formed by certain semigroups $M_{k}(S, \hbar)$ with $k \geq 1, S \in \mathbf{V}, A$ an alphabet and $\hbar: A^{+} \rightarrow S$ a $k$ superposition homomorphism. Therefore, possible properties of $\mathbf{V} * \mathbf{D}$ may be tested on the semigroups $M_{k}(S, \hbar)$ and Almeida and Azevedo applied those test-semigroups (an expression used in [1]) to obtain a representation of the free pro- $(\mathbf{V} * \mathbf{D})$ semigroup over $A$.

In this paper, we introduce a class of local groups $\mathcal{S}(G, L, \mathrm{f})$ with $G$ a group, $L \subseteq A^{+}$a factorial language and $\mathrm{f}: L \cup \ddot{L} \rightarrow G$ a function where, informally speaking, $\ddot{L}$ is formed by the words over $A$ with minimal length that do not belong to $L$. The group $G$ is the maximal subgroup of $\mathcal{S}(G, L, \mathrm{f}), L$ is the set of non-regular elements of $\mathcal{S}(G, L, \mathrm{f})$ and f serves to define the operation of the semigroup. Given a semigroup presentation for $G$, we describe a semigroup presentation for $\mathcal{S}(G, L, f)$. In particular, when $G$ is finitely presented, the semigroup presentation for $\mathcal{S}(G, L, \mathrm{f})$ is finite if and only if $L$ is finite. For a finite group $G$, the semigroup $M_{k}(G, \hbar)$ is a local group. We show that, when $L_{k}$ is the language of all words over $A$ of length at most $k$ and $\mathrm{f}$ is the restriction of $\hbar$ to $L \cup \ddot{L}, M_{k}(G, \hbar)$ is a subsemigroup of a homomorphic image of $S_{k}(G, \mathrm{f})=\mathcal{S}\left(G, L_{k}, \mathrm{f}\right)$. As a consequence of the above mentioned results of Almeida and Azevedo, we deduce that the semigroups $S_{k}(G, \mathrm{f})$, with $k \geq 1$ and $G \in \mathbf{H}$, form a generating set of the pseudovariety $\mathbf{L H}$. Obviously, the more general class of semigroups $\mathcal{S}(G, L, \mathrm{f})$, with $G \in \mathbf{H}$ and $L$ finite, generates $\mathbf{L H}$. While the semigroups $S_{k}(G, \mathrm{f})$ and $M_{k}(G, \hbar)$ have essentially the same capabilities as test-semigroups for $\mathbf{L H}$, the semigroups $\mathcal{S}(G, L, \mathrm{f})$ have the advantage that one may explore the possibility of choosing appropriate languages and functions $\mathrm{f}$ to test specific properties of $\mathbf{L H}$. This makes the semigroups $\mathcal{S}(G, L, \mathrm{f})$ interesting alternatives to the semigroups $M_{k}(G, \hbar)$.

We will use the semigroups $\mathcal{S}(G, L, \mathbf{f})$ to show that $\mathbf{L G}$ and $\mathbf{S}$, the pseudovariety of all finite semigroups, satisfy the same identities involving $\kappa$-terms of rank at most 1 (i.e., terms obtained from ones of the form $u$ and $v^{\omega-1}$, with $u \in A^{*}$ and $v \in A^{+}$, by finite concatenation) and that these identities are decidable over $\mathbf{L G}$ (and $\mathbf{S}$ ). The semigroups $\mathcal{S}(G, L, \mathrm{f}$ ) will be employed in a more general context in [7] to solve the word problem for identities involving arbitrary $\kappa$-terms over LG. Recall that this type of word problem is already solved, for instance, 
for the pseudovarieties LI of locally trivial semigroups [1] and LSl of local semilattices [4. Althought the pseudovariety $\mathbf{S l}$ of finite semilattices is not a pseudovariety of groups, the equality $\mathbf{L S l}=\mathbf{S l} * \mathbf{D}$ holds and the representations of free pro- $(\mathbf{S l} * \mathbf{D})$ semigroups, obtained by Almeida and Azevedo, were used to solve the above mentioned word problem as well as to prove other properties of LSI [8, 6$]$.

\section{Semigroup presentations for a class of local groups}

In this section we introduce a certain type of semigroup presentation and prove that the semigroups they define are local groups. For an introduction to combinatorics on words, the reader is referred to [11].

We begin by giving a characterization of local groups. Recall that a semigroup is simple if it has a unique ideal. The set of idempotents of a semigroup $S$, denoted by $E(S)$, is endowed with a natural (partial) order relation defined by the rule that $e \leq f$ if and only if $e f=f e=e$. A simple semigroup $S$ is said to be completely simple if $E(S)$ has minimal elements for the relation $\leq$.

Proposition 2.1 A semigroup $S$ is a local group if and only if $E(S)=\emptyset$ or $S$ has a completely simple minimal ideal containing $E(S)$.

Proof. Let $S$ be a local group and suppose that $E(S)$ is non-empty. Denote by $J_{a}$ the principal ideal $S^{1} a S^{1}$ generated by an element $a$ of $S$. Let $e \in E(S)$. Then, by definition of local group, the local submonoid $e S e$ is a group with identity $e$. Hence, for each $a \in S$ there exists $a^{\prime} \in S$ such that $(e a e)\left(e a^{\prime} e\right)=e$. This means that, for all $a \in S, e \in J_{a}$ and so $J_{e} \subseteq J_{a}$. Since $e$ is an arbitrary idempotent, it follows that $J=J_{e}$ is a minimal ideal of $S$ containing $E(S)$. We now claim that every element of $E(S)$ is minimal for the relation $\leq$. Indeed, let $e, f \in E(S)$ and suppose that $e \leq f$, that is, suppose that $e f=f e=e$. Then $f e f=e$, whence $e$ belongs to the group $f S f$ with identity $f$. The fact that $e$ is an idempotent shows that $e=f$ and proves the claim. We conclude that $J$ is a completely simple semigroup.

Reciprocally, if $E(S)=\emptyset$ then $S$ is (trivially) a local group. Suppose now that $E(S)$ is non-empty, that $S$ has a completely simple minimal ideal $J$ and that $E(S)$ is a subset of $J$. In particular, by the Rees-Suschkewitsch Theorem, $J$ is isomorphic to a Rees matrix semigroup over a group $G$. Moreover, since all the idempotents of $S$ are in $J$, one deduces that each local submonoid $e S e$ is $H_{e}$, the $\mathcal{H}$-class of $e$, a group isomorphic to $G$, thus proving that $S$ is a local group.

Consider a word $w=a_{1} a_{2} \ldots a_{n} \in A^{+}\left(a_{i} \in A\right)$ of length $n \geq 1$. For $1 \leq p \leq q \leq n$, we denote $w[p, q]=a_{p} a_{p+1} \cdots a_{q}$. A word $u \in A^{+}$is a (non-empty) factor of $w$ if $u=w[p, q]$ for some $p$ and $q$. In this case $w[p, q]$ is said to be an occurrence of the factor $u$ in $w$. We will say also "an occurrence $u=w[p, q]$ in $w$ " instead of "an occurrence $w[p, q]$ of $u$ in $w$ ". If there is an occurrence $u=w[p, q]$ with $p=1$ (resp. $q=n$ ), then $u$ is called a prefix (resp. a suffix) of 
$w$. We denote by $w_{\alpha}$ and $w_{\omega}$, respectively, the prefix $w[1, n-1]$ and the suffix $w[2, n]$ of $w$ of length $n-1$. A language $L \subseteq A^{+}$is said to be factorial if it is closed under taking non-empty factors. Let $L$ be a non-empty factorial language and let

$$
\ddot{L}=\left\{v \in A^{+}: v \notin L \text { and } v_{\alpha}, v_{\omega} \in L\right\} .
$$

We assume that $L$ has content $A$ (i.e., $A \subseteq L$ ) and observe that $L$ and $\ddot{L}$ are disjoint languages. Observe also the following elementary facts

$$
\begin{array}{cc}
\forall v_{1}, v_{2} \in \ddot{L} \quad\left(v_{1} \text { is a factor of } v_{2} \Leftrightarrow v_{1}=v_{2}\right) ; \\
\forall w \in A^{+} \quad(\ddot{L} \cap F(w)=\emptyset \Leftrightarrow w \in L) .
\end{array}
$$

From (2.2) it follows that $\ddot{L}=\emptyset$ if and only if $L=A^{+}$. We associate to any given word $w=a_{1} a_{2} \ldots a_{n} \in A^{+}\left(a_{i} \in A\right)$ a well-determined finite sequence

$$
\operatorname{sc}_{L}[w]=\left(w_{0}, \ddot{w}_{1}, w_{1}, \ldots, \ddot{w}_{m}, w_{m}\right)
$$

called the sequence of coordinates of $w$ determined by $L$, as follows:

- $m \geq 0$ is the number, called the $\ddot{L}$-length of $w$, of occurrences of elements of $\ddot{L}$ in $w$. Observe that $m=0$ if and only if $w \in L$, in which case $\operatorname{sc}_{L}[w]=\left(w_{0}\right)$.

- if $m>0$ then $\ddot{w}_{1}=w\left[p_{1}, q_{1}\right], \ldots, \ddot{w}_{m}=w\left[p_{m}, q_{m}\right]$ are the successive occurrences of factors of $w$ that belong to $\ddot{L}$. Notice that, for every $i, p_{i}<q_{i}$ and, by (2.1), the integer interval $\left[p_{i}, \ldots, q_{i}\right]$ is contained in $\left[p_{j}, \ldots, q_{j}\right]$ for some $j$ if and only if $i=j$.

- if $m>0$ then $w_{0}=w\left[1, q_{1}-1\right], w_{m}=w\left[p_{m}+1, n\right]$ and $w_{i}=w\left[p_{i}+1, q_{i+1}-1\right]$ for $0<i<m$. We note that $w_{0} a_{q_{1}}=w\left[1, q_{1}\right]$ does not belong to $L$ (since neither does $w\left[p_{1}, q_{1}\right]$ and $L$ is factorial), whence $w_{0}$ is the longest prefix of $w$ in $L$. Analogously, $w_{m}$ is the longest suffix of $w$ that belongs to $L$. Moreover, for $0<i<m$, the factors $w_{i} a_{q_{i+1}}=w\left[p_{i}+1, q_{i+1}\right]$ and $a_{p_{i}} w_{i}=w\left[p_{i}, q_{i+1}-1\right]$ do not belong to $L$. We then say that $w_{0}, w_{1}, \ldots, w_{m}$ are maximal factors of $w$ in $L$. With this sense of maximality, one may verify that $w_{0}, w_{1}, \ldots, w_{m}$ is the sequence of all maximal factors of $w$ in $L$ by the order they occur in $w$.

Alternatively, the sequence $\operatorname{sc}_{L}[w]$ may be constructed by the recursive application of the two following steps:

- If $w \in L$ then let $\operatorname{sc}_{L}[w]=(w)$.

- If $w \notin L$ then select an occurrence $\ddot{v}=w[p, q]$ in $w$ of a factor $\ddot{v} \in \ddot{L}$ and $\operatorname{let} \operatorname{sc}_{L}[w]=$ $\operatorname{sc}_{L}\left[z_{1}\right](\ddot{v}) \operatorname{sc}_{L}\left[z_{2}\right]$, where $z_{1}=w[1, q-1]$ and $z_{2}=w[p+1, n]$. 
Example 2.2 For $A=\{a, b\}$, consider the factorial languages $L_{1}=\left\{a, b, a^{2}, a b, b^{2}, a^{3}, a^{2} b\right\}$ and $L_{2}=\left\{a^{i}, a^{j} b a^{k}: i>0, j \geq 0, k \geq 0\right\}$ over $A$. Then $\ddot{L}_{1}=\left\{b a, a b^{2}, b^{3}, a^{4}, a^{3} b\right\}$ and $\ddot{L}_{2}=\left\{b a^{j} b: j \geq 0\right\}$. For the words $u=a^{5} b a^{2}$ and $v=a b^{3} a b a^{4} b$, we have

$$
\begin{aligned}
& s c_{L_{1}}[u]=\left(a^{3}, a^{4}, a^{3}, a^{4}, a^{3}, a^{3} b, a^{2} b, b a, a^{2}\right), \\
& s c_{L_{2}}[u]=(u), \\
& s c_{L_{1}}[v]=\left(a b, a b^{2}, b^{2}, b^{3}, b^{2}, b a, a b, b a, a^{3}, a^{4}, a^{3}, a^{3} b, a^{2} b\right), \\
& s c_{L_{2}}[v]=\left(a b, b^{2}, b, b^{2}, b a, b a b, a b a^{4}, b a^{4} b, a^{4} b\right) .
\end{aligned}
$$

The above construction may be reverted. More precisely, given $\operatorname{sc}_{L}[w]$ there is a deterministic procedure to calculate the word $w$. This shows in particular that

$$
\forall w, z \in A^{+}\left(\operatorname{sc}_{L}[w]=\operatorname{sc}_{L}[z] \Leftrightarrow w=z\right)
$$

The procedure is the following. If $\operatorname{sc}_{L}[w]=\left(w_{0}\right)$, then $w=w_{0}$. Suppose now that $\operatorname{sc}_{L}[w]=$ $\left(w_{0}, \ddot{w}_{1}, w_{1}, \ldots, \ddot{w}_{m}, w_{m}\right)$ with $m \geq 1$ and assume that is possible to determine a word $z$ of $\ddot{L}$ length $m-1$ given its sequence of coordinates $\operatorname{sc}_{L}[z]$. By the above construction, $w_{0}=w^{\prime}\left(\ddot{w}_{1}\right)_{\alpha}$ for some $w^{\prime} \in A^{*}$ and the sequence $\left(w_{1}, \ddot{w}_{2}, \ldots, \ddot{w}_{m}, w_{m}\right)$ is precisely $\operatorname{sc}_{L}[z]$ where $z=\left(\ddot{w}_{1}\right)_{\omega} w^{\prime \prime}$ is such that $w=w^{\prime} \ddot{w}_{1} w^{\prime \prime}$. Since $w_{0}$ and $\ddot{w}_{1}$ are given, we may determine $w^{\prime}$. On the other hand, by hypothesis, $z$ is calculable and, so, also is $w^{\prime \prime}$. Therefore $w$ is calculable.

Let $\left\langle A_{G} \mid R_{G}\right\rangle$ be a semigroup presentation for a group $G$, so that $G \cong A_{G}^{+} / \rho_{R_{G}}$. For simplicity of notation, we will usually regard a given word $w \in A_{G}^{+}$as the element of $G$ it represents. On the other hand, by choosing for each element $g \in G$ a word of $A_{G}^{+}$representing $g$ we may view $G$ as a subset of $A_{G}^{+}$. In particular, we denote by $e$ a word of $A_{G}^{+}$representing $1_{G}$, whence

$$
\forall w \in A_{G}^{+}, w=e w=w e \text { in } G .
$$

Let $X=A \cup A_{G}$, let $L^{1}=L \cup\{1\}$ and let $\mathrm{f}: L \cup \ddot{L} \rightarrow G$ be a function. We associate to $\mathrm{f}$ four new functions $\check{\mathrm{f}}: A^{+} \rightarrow L \cup L G L, \dot{\mathrm{f}}: A^{*} \rightarrow L^{1} \cup G L, \grave{\mathrm{f}}: A^{*} \rightarrow L^{1} \cup L G$ and $\hat{\mathrm{f}}: A^{*} \rightarrow G$ defined as follows: $\mathfrak{f}(1)=\grave{f}(1)=1$ and $\hat{\mathbf{f}}(1)=1_{G}$; if $w \in L$ then $\check{\mathbf{f}}(w)=\grave{f}(w)=\grave{\mathbf{f}}(w)=w$ and $\hat{\mathbf{f}}(w)=\mathbf{f}(w)$; if $w \in A^{+} \backslash L$ and $\operatorname{sc}_{L}[w]=\left(w_{0}, \ddot{w}_{1}, w_{1}, \ldots, \ddot{w}_{m}, w_{m}\right)$, then

$$
\begin{aligned}
& \check{\mathbf{f}}(w)=w_{0} \mathbf{f}\left(\ddot{w}_{1}\right) \mathbf{f}\left(w_{1}\right) \mathbf{f}\left(\ddot{w}_{2}\right) \cdots \mathrm{f}\left(\ddot{w}_{m}\right) w_{m}, \\
& \hat{f}(w)=\mathbf{f}\left(w_{0}\right) \mathbf{f}\left(\ddot{w}_{1}\right) \mathbf{f}\left(w_{1}\right) \mathbf{f}\left(\ddot{w}_{2}\right) \cdots \mathbf{f}\left(\ddot{w}_{m}\right) w_{m}, \\
& \grave{f}(w)=w_{0} \mathbf{f}\left(\ddot{w}_{1}\right) \mathbf{f}\left(w_{1}\right) \mathbf{f}\left(\ddot{w}_{2}\right) \cdots \mathrm{f}\left(\ddot{w}_{m}\right) \mathbf{f}\left(w_{m}\right), \\
& \hat{\mathrm{f}}(w)=\mathrm{f}\left(w_{0}\right) \mathrm{f}\left(\ddot{w}_{1}\right) \mathrm{f}\left(w_{1}\right) \mathrm{f}\left(\ddot{w}_{2}\right) \cdots \mathrm{f}\left(\ddot{w}_{m}\right) \mathrm{f}\left(w_{m}\right) \text {. }
\end{aligned}
$$

Now, we let $\check{\mathrm{f}}: X^{+} \rightarrow L \cup L^{1} G L^{1}$ be the extension to $X^{+}$of the above function $\check{\mathrm{f}}$ by setting $\check{\mathrm{f}}(w)=\grave{\mathrm{f}}\left(u_{0}\right) g_{1} \hat{\mathrm{f}}\left(u_{1}\right) \cdots g_{n-1} \hat{\mathrm{f}}\left(u_{n-1}\right) g_{n} \hat{\mathrm{f}}\left(u_{n}\right)$ for each word $w=u_{0} g_{1} u_{1} \cdots g_{n} u_{n} \in X^{+} \backslash A^{+}$, with $u_{0}, u_{n} \in A^{*}, u_{1}, \ldots, u_{n-1} \in A^{+}$and $g_{1}, \ldots, g_{n} \in A_{G}^{+}$.

It is worth observing the following properties of the functions $\hat{f}$ and $\check{f}$.

Lemma 2.3 Let $w, z \in A^{+}$and let $z_{0} \in L$ be the first coordinate of $z$ and $w_{m} \in L$ be the last coordinate of $w$ determined by $L$. Then, 
(a) $\hat{\mathbf{f}}(w z)=\hat{\mathbf{f}}\left(w z_{0}\right) \mathfrak{f}\left(z_{0}\right)^{-1} \hat{\mathbf{f}}(z)=\hat{\mathbf{f}}(w) \mathbf{f}\left(w_{m}\right)^{-1} \hat{\mathbf{f}}\left(w_{m} z\right)$;

(b) $\check{\mathfrak{f}}(w z)=\check{\mathbf{f}}(\check{\mathrm{f}}(w) \check{\mathrm{f}}(z))$.

Proof. Let

$$
\begin{aligned}
\operatorname{sc}_{L}[w] & =\left(w_{0}, \ddot{w}_{1}, w_{1}, \ldots, \ddot{w}_{m}, w_{m}\right), \\
\operatorname{sc}_{L}[z] & =\left(z_{0}, \ddot{z}_{1}, z_{1}, \ldots, \ddot{z}_{n}, z_{n}\right), \\
x & =w_{m} z_{0}, \\
\operatorname{sc}_{L}[x] & =\left(x_{0}, \ddot{x}_{1}, x_{1}, \ldots, \ddot{x}_{p}, x_{p}\right) .
\end{aligned}
$$

Hence

$$
\begin{aligned}
\operatorname{sc}_{L}\left[w z_{0}\right] & =\left(w_{0}, \ddot{w}_{1}, w_{1}, \ldots, \ddot{w}_{m}, x_{0}, \ddot{x}_{1}, x_{1}, \ldots, \ddot{x}_{p}, x_{p}\right) \\
\operatorname{sc}_{L}[w z] & =\left(w_{0}, \ddot{w}_{1}, w_{1}, \ldots, \ddot{w}_{m}, x_{0}, \ddot{x}_{1}, x_{1}, \ldots, \ddot{x}_{p}, x_{p}, \ddot{z}_{1}, z_{1}, \ldots, \ddot{z}_{n}, z_{n}\right) .
\end{aligned}
$$

Then,

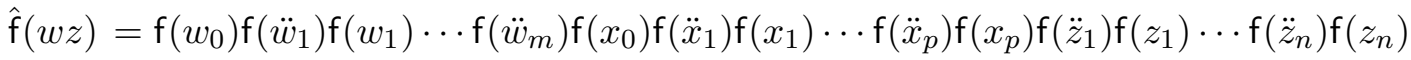

$$
\begin{aligned}
& =\hat{\mathbf{f}}\left(w z_{0}\right) \mathbf{f}\left(z_{0}\right)^{-1} \mathbf{f}\left(z_{0}\right) \mathbf{f}\left(\ddot{z}_{1}\right) \mathbf{f}\left(z_{1}\right) \cdots \mathbf{f}\left(\ddot{z}_{n}\right) \mathbf{f}\left(z_{n}\right) \\
& =\hat{\mathbf{f}}\left(w z_{0}\right) \mathbf{f}\left(z_{0}\right)^{-1} \hat{\mathbf{f}}(z) \text {. }
\end{aligned}
$$

One can show analogously that $\hat{\mathbf{f}}(w z)=\hat{\mathrm{f}}(w) \mathrm{f}\left(w_{m}\right)^{-1} \hat{\mathbf{f}}\left(w_{m} z\right)$, thus concluding the proof of $(a)$,

If $m=n=0$ then $w, z \in L$ and (b) is trivially verified. When $m, n>0$, we have

$$
\begin{aligned}
\check{\mathrm{f}}(\check{\mathrm{f}}(w) \check{\mathrm{f}}(z)) & =\check{\mathrm{f}}\left(w_{0} \mathrm{f}\left(\ddot{w}_{1}\right) \mathrm{f}\left(w_{1}\right) \cdots \mathrm{f}\left(\ddot{w}_{m}\right) w_{m} z_{0} \mathrm{f}\left(\ddot{z}_{1}\right) \mathrm{f}\left(z_{1}\right) \cdots \mathrm{f}\left(\ddot{z}_{n}\right) z_{n}\right) \\
& =w_{0} \mathrm{f}\left(\ddot{w}_{1}\right) \mathrm{f}\left(w_{1}\right) \cdots \mathrm{f}\left(\ddot{w}_{m}\right) \hat{\mathrm{f}}\left(w_{m} z_{0}\right) \mathrm{f}\left(\ddot{z}_{1}\right) \mathrm{f}\left(z_{1}\right) \cdots \mathrm{f}\left(\ddot{z}_{n}\right) z_{n} \\
& =w_{0} \mathrm{f}\left(\ddot{w}_{1}\right) \mathrm{f}\left(w_{1}\right) \cdots \mathrm{f}\left(\ddot{w}_{m}\right) \mathrm{f}\left(x_{0}\right) \mathrm{f}\left(\ddot{x}_{1}\right) \mathrm{f}\left(x_{1}\right) \cdots \mathrm{f}\left(\ddot{x}_{p}\right) \mathrm{f}\left(x_{p}\right) \mathrm{f}\left(\ddot{z}_{1}\right) \mathrm{f}\left(z_{1}\right) \cdots \mathrm{f}\left(\ddot{z}_{n}\right) z_{n} \\
& =\check{f}(w z) .
\end{aligned}
$$

If $m=0$ and $n>0$ then $\operatorname{sc}_{L}[w z]=\left(x_{0}, \ddot{x}_{1}, x_{1}, \ldots, \ddot{x}_{p}, x_{p}, \ddot{z}_{1}, z_{1}, \ldots, \ddot{z}_{n}, z_{n}\right)$ and the equality $\check{\mathrm{f}}(w z)=\check{\mathrm{f}}(\check{\mathrm{f}}(w) \check{\mathrm{f}}(z))$ is checked as above. The case $m>0$ and $n=0$ is symmetric.

For latter reference, we state the following extension of Lemma $2.3(b)$,

$$
\forall w, z \in X^{+}, \check{\mathrm{f}}(w z)=\check{\mathrm{f}}(\check{\mathrm{f}}(w) \check{\mathrm{f}}(z)),
$$

whose validity may be verified by the reader.

We finally set up the presentations for our local groups. For each $u \in L$ and $\ddot{v} \in \ddot{L}$, we define the following relations over $X$

$$
\begin{aligned}
r_{u}: & \text { eue } & =\mathrm{f}(u) \\
r_{\ddot{v}}: & \ddot{v} & =\ddot{v}_{\alpha} \mathrm{f}(\ddot{v}) \ddot{v}_{\omega}
\end{aligned}
$$

and set $R_{\mathrm{f}}=\left\{r_{u}, r_{\ddot{v}}: u \in L, \ddot{v} \in \ddot{L}\right\}$. Denote by $\mathcal{T}[G, L, \mathrm{f}]$ the semigroup $T$ defined by the presentation $\langle X \mid R\rangle$, where $R=R_{G} \cup R_{\mathrm{f}}$. Taking (2.4) into account, it can be shown that the following relations hold in $T$ for any $w \in A^{+}$and $z \in A^{+} \backslash L$

$$
e \check{\mathrm{f}}(w) e=\hat{\mathrm{f}}(w), \quad e \check{\mathrm{f}}(z)=\grave{\mathbf{f}}(z), \quad \check{\mathrm{f}}(z) e=\grave{\mathbf{f}}(z) .
$$

A more important relation valid in $T$ is revealed in the next lemma. 
Lemma 2.4 If $w \in X^{+}$is an arbitrary word, then $w=\check{\mathrm{f}}(w)$ in $T$.

Proof. We consider first the case where $w \in A^{+}$and let $\operatorname{sc}_{L}[w]=\left(w_{0}, \ddot{w}_{1}, w_{1}, \ldots, \ddot{w}_{m}, w_{m}\right)$. We prove the result by induction on the $\ddot{L}$-length $m$ of $w$. If $m=0$, then $w=w_{0} \in L$ and so $\check{\mathrm{f}}(w)=w$ by definition of $\check{\mathrm{f}}$. Suppose now that $m \geq 1$ and assume, by induction hypothesis, that the result is valid for words $z \in A^{+}$with $\ddot{L}$-length $m-1$. Write $w=w^{\prime} \ddot{w}_{1} w^{\prime \prime}$ where the first occurrence of $\ddot{w}_{1}$ is distinguished in the factorization. Applying relation $r_{\ddot{w}_{1}}$ to that occurrence of $\ddot{w}_{1}$ one deduces that $T$ verifies $w=w^{\prime}\left(\ddot{w}_{1}\right)_{\alpha} \mathrm{f}\left(\ddot{w}_{1}\right)\left(\ddot{w}_{1}\right)_{\omega} w^{\prime \prime}$. Moreover $w_{0}=w^{\prime}\left(\ddot{w}_{1}\right)_{\alpha}$ and $\operatorname{sc}_{L}[z]=\left(w_{1}, \ddot{w}_{2}, w_{2}, \ldots, \ddot{w}_{m}, w_{m}\right)$ where $z=\left(\ddot{w}_{1}\right)_{\omega} w^{\prime \prime}$, whence $T$ satisfies $w=w_{0} \mathrm{f}\left(\ddot{w}_{1}\right) z$. If $m=1$, then $z=w_{1}$ and thus $T$ verifies $w=\check{\mathfrak{f}}(w)$. Suppose next that $m>1$ and notice that, by induction hypothesis, $z=\check{\mathrm{f}}(z)$ in $T$. So, $T$ verifies $w=w_{0} \mathrm{f}\left(\ddot{w}_{1}\right) \check{\mathrm{f}}(z)=w_{0} \mathrm{f}\left(\ddot{w}_{1}\right) w_{1} \mathrm{f}\left(\ddot{w}_{2}\right) \mathrm{f}\left(w_{2}\right) \mathrm{f}\left(\ddot{w}_{3}\right) \cdots \mathrm{f}\left(\ddot{w}_{m}\right) w_{m}$. Then, by (2.4), the semigroup $T$ verifies $w=w_{0} f\left(\ddot{w}_{1}\right) e w_{1} e f\left(\ddot{w}_{2}\right) f\left(w_{2}\right) \cdots f\left(\ddot{w}_{m}\right) w_{m}$ and also, applying relation $r_{w_{1}}$, $w=w_{0} \mathbf{f}\left(\ddot{w}_{1}\right) \mathbf{f}\left(w_{1}\right) \mathbf{f}\left(\ddot{w}_{2}\right) \mathbf{f}\left(w_{2}\right) \cdots \mathfrak{f}\left(\ddot{w}_{m}\right) w_{m}=\check{\mathfrak{f}}(w)$, thus concluding the inductive step and the proof of the lemma for $w \in A^{+}$.

Suppose now that $w=u_{0} g_{1} u_{1} \cdots g_{n} u_{n} \in X^{+} \backslash A^{+}$, with $u_{0}, u_{n} \in A^{*}, u_{1}, \ldots, u_{n-1} \in A^{+}$ and $g_{1}, \ldots, g_{n} \in A_{G}^{+}$. So, in $T$,

$$
\begin{aligned}
& w=\check{\mathfrak{f}}\left(u_{0}\right) g_{1} \check{\mathrm{f}}\left(u_{1}\right) g_{2} \check{\mathrm{f}}\left(u_{2}\right) \cdots g_{n} \check{\mathrm{f}}\left(u_{n}\right) \quad \text { by the first case } \\
& =\check{\mathrm{f}}\left(u_{0}\right) e g_{1} e \check{\mathrm{f}}\left(u_{1}\right) e g_{2} e \check{\mathrm{f}}\left(u_{2}\right) \cdots e g_{n} e \check{\mathrm{f}}\left(u_{n}\right) \quad \text { by (2.4) } \\
& =\grave{f}\left(u_{0}\right) g_{1} \hat{\mathrm{f}}\left(u_{1}\right) g_{2} \hat{\mathrm{f}}\left(u_{2}\right) \cdots g_{n} \hat{\mathrm{f}}\left(u_{n}\right) \quad \text { by (2.6) } \\
& =\check{\mathrm{f}}(w) \text {. }
\end{aligned}
$$

This concludes the proof of the lemma.

Let $\psi$ be the canonical epimorphism from $X^{+}$onto $X^{+} / \rho_{R}=T$ and denote by $Z$ the subset $L \cup L^{1} G L^{1}$ of $X^{+}$. By Lemma 2.4, the word $\check{\mathrm{f}}(w) \in Z$ is a representative of the element $\psi(w) \in T$. We show next that $Z$ contains exactly one representative of each element of $T$ and endow $Z$ with a (natural) structure of semigroup that makes it isomorphic to $T$. Before that we mention that, obviously, $Z=\check{\mathrm{f}}\left(X^{+}\right)$and $\check{\mathrm{f}}(z)=z$ for every $z \in Z$, whence $\check{\mathrm{f}} \circ \check{\mathrm{f}}=\check{\mathrm{f}}$.

Proposition 2.5 Let $Z$ be endowed with the operation defined by $z_{1} \cdot z_{2}=\check{\mathrm{f}}\left(z_{1} z_{2}\right)$ and let $J$ be the Rees matrix semigroup $\mathcal{M}\left[G ; L^{1}, L^{1} ; P\right]$ where $P=(\hat{\mathrm{f}}(u v))_{u, v \in L^{1}}$.

(a) The operation - is associative (and we denote by $\mathcal{Z}[G, L, \mathrm{f}]$ the semigroup $Z$ ).

(b) The mapping $\check{\mathrm{f}}: X^{+} \rightarrow Z$ is an epimorphism.

(c) For every $y, w \in X^{+}, \check{\mathrm{f}}(y)=\check{\mathrm{f}}(w)$ if and only if $y=w$ in $T$.

(d) The semigroup $Z$ is isomorphic to $T$.

(e) $Z$ is a local group with minimal ideal $I=L^{1} G L^{1}$ isomorphic to $J$. 
Proof. In order to verify $(a)$, let $z_{1}, z_{2}, z_{3} \in Z$ and notice that $z_{1} \cdot\left(z_{2} \cdot z_{3}\right)=\check{\mathrm{f}}\left(z_{1} \check{\mathrm{f}}\left(z_{2} z_{3}\right)\right)$ by definition of $\cdot$. Now, as $z_{1} \in Z$, we have $z_{1}=\check{\mathrm{f}}\left(z_{1}\right)$ and so, by (2.5),$z_{1} \cdot\left(z_{2} \cdot z_{3}\right)=\check{\mathrm{f}}\left(z_{1} z_{2} z_{3}\right)$. By symmetry $\left(z_{1} \cdot z_{2}\right) \cdot z_{3}=\check{\mathrm{f}}\left(z_{1} z_{2} z_{3}\right)$ which shows the associativity of $\cdot$

Let $y, w \in X^{+}$. Then $\check{\mathfrak{f}}(y)$ and $\check{\mathrm{f}}(w)$ belong to $Z$ and, so, by definition of $\cdot$ and by (2.5), $\check{\mathfrak{f}}(y) \cdot \check{\mathbf{f}}(w)=\check{\mathbf{f}}(\check{\mathfrak{f}}(y) \check{\mathbf{f}}(w))=\check{\mathbf{f}}(y w)$. Since $\check{\mathbf{f}}$ is clearly onto, $(b)$ is proved.

For $(c)$, suppose first that $\check{\mathrm{f}}(y)=\check{\mathrm{f}}(w)$. By Lemma 2.4, $y=\check{\mathrm{f}}(y)$ and $w=\check{\mathrm{f}}(w)$ in $T$, whence also $y=w$ in $T$. Suppose next that $y=w$ in $T$. Without loss of generality we may assume that $w$ is deduced from $y$ in one step, so that $y=y^{\prime} x_{1} y^{\prime \prime}$ and $w=y^{\prime} x_{2} y^{\prime \prime}$ with $\left(x_{1}=x_{2}\right) \in R$. Since by $(b)$ 解 is a homomorphism, to deduce $\check{\mathrm{f}}(y)=\check{\mathrm{f}}(w)$ it suffices to show that $\check{\mathrm{f}}\left(x_{1}\right)=\check{\mathrm{f}}\left(x_{2}\right)$. If $\left(x_{1}=x_{2}\right) \in R_{G}$ then $x_{1}, x_{2} \in A_{G}^{+}$and $x_{1}=x_{2}$ in $G$. Therefore, if $x \in G$ represents both $x_{1}$ and $x_{2}$, then $\check{\mathrm{f}}\left(x_{1}\right)=x=\check{\mathrm{f}}\left(x_{2}\right)$. Suppose next that $x_{1}=x_{2}$ is the relation $r_{u} \in R_{\mathrm{f}}$, that is eue $=\mathrm{f}(u)$, for some $u \in L$. We have $\check{\mathrm{f}}($ eue $)=e \hat{\mathrm{f}}(u) e$ by definition of $\check{\mathrm{f}}$. As $u \in L, \hat{\mathrm{f}}(u)=\mathrm{f}(u)$. Since $e$ represents the identity of $G$, ef $(u) e=\mathrm{f}(u)$ in $G$ and so $\check{f}(e u e)=\mathrm{f}(u)=\check{\mathrm{f}}(\mathrm{f}(u))$. It remains to treat the case where $x_{1}=x_{2}$ is the relation $r_{\ddot{v}}$, that is $\ddot{v}=\ddot{v}_{\alpha} \mathrm{f}(\ddot{v}) \ddot{v}_{\omega}$, of $R_{\mathrm{f}}$ for some $\ddot{v} \in \ddot{L}$. If $\ddot{v} \in \ddot{L}$ then $\operatorname{sc}_{L}[\ddot{v}]=\left(\ddot{v}_{\alpha}, \ddot{v}, \ddot{v}_{\omega}\right)$ and so $\check{f}(\ddot{v})=\ddot{v}_{\alpha} \mathrm{f}(\ddot{v}) \ddot{v}_{\omega}$. Now, $\ddot{v}_{\alpha} \mathrm{f}(\ddot{v}) \ddot{v}_{\omega}=\check{\mathrm{f}}\left(\ddot{v}_{\alpha} \mathrm{f}(\ddot{v}) \ddot{v}_{\omega}\right)$ because $\ddot{v}_{\alpha} \mathrm{f}(\ddot{v}) \ddot{v}_{\omega} \in Z$, whence $\check{\mathrm{f}}(\ddot{v})=\check{\mathrm{f}}\left(\ddot{v}_{\alpha} \mathrm{f}(\ddot{v}) \ddot{v}_{\omega}\right)$. This completes the proof of $(c)$.

To deduce $(d)$ it suffices to notice that the existence of an isomorphism $\theta: T \rightarrow Z$ is an immediate consequence of $(b)$ and $(c)$. It is the unique mapping from $T$ onto $Z$ such that $\theta \circ \psi=\check{\mathrm{f}}$, where $\psi$ is the canonical epimorphism from $X^{+}$onto $T$.

It is not difficult to verify that $I=L^{1} G L^{1}$ is the minimal ideal of $Z$ and that $\varphi: I \rightarrow$ $J$, ugv $\mapsto(u, g, v)$, defines an isomorphism from $I$ onto $J$. Moreover, the Rees quotient $Z / I=L \cup\{0\}$ is a nilpotent semigroup (i.e., $Z / I$ has 0 as its unique idempotent). Hence, by Proposition 2.1, $Z$ is a local group and this finishes the proof of the proposition.

Let $S=L \cup J=L \cup\left(L^{1} \times G \times L^{1}\right)$. We extend the mapping $\varphi: I \rightarrow J$ above to a new one $\varphi: Z \rightarrow S$ by setting $\varphi(w)=w$ for every $w \in L$. Next, we define an operation $\odot$ in $S$ by setting, for every $w, w^{\prime} \in L$ and $(u, g, v),\left(u^{\prime}, g^{\prime}, v^{\prime}\right) \in J$,

$$
\begin{aligned}
w \odot w^{\prime} & =\varphi\left(w \cdot w^{\prime}\right), & w \odot(u, g, v) & =\varphi(w \cdot u g v), \\
(u, g, v) \odot w & =\varphi(u g v \cdot w), & (u, g, v) \odot\left(u^{\prime}, g^{\prime}, v^{\prime}\right) & =\varphi\left(u g v \cdot u^{\prime} g^{\prime} v^{\prime}\right) .
\end{aligned}
$$

As one may verify, this operation makes $S$ a semigroup, which we denote by $\mathcal{S}[G, L, \mathrm{f}]$, and $\varphi$ an isomorphism from $Z$ onto $S$. The following statement is then an immediate consequence of previous results.

Corollary 2.6 Let $L \subseteq A^{+}$be a non-empty factorial language, let $G$ be a group defined by a presentation $\left\langle A_{G} \mid R_{G}\right\rangle$ and let $\mathrm{f}: L \cup \ddot{L} \rightarrow G$ be a function. The semigroups $\mathcal{S}[G, L, \mathrm{f}]$, $\mathcal{T}[G, L, \mathrm{f}]$ and $\mathcal{Z}[G, L, \mathrm{f}]$ are isomorphic local groups, defined by the presentation $\left\langle A \cup A_{G}\right|$ $\left.R_{G} \cup R_{\mathrm{f}}\right\rangle$, with minimal ideal $\mathcal{M}\left[G ; L^{1}, L^{1} ; P\right]$, where $P=(\hat{\mathfrak{f}}(u v))_{u, v \in L^{1}}$, and $L$ as set of nonregular elements. Therefore, the above semigroups are finite if and only if both $L$ and $G$ are finite. 


\section{Generators for $\mathrm{LH}$}

In this section, we let $\mathbf{H}$ denote a pseudovariety of groups and show that the semigroups $\mathcal{S}[G, L, \mathrm{f}]$, with $G \in \mathbf{H}$ and $L$ finite, form a generating set of the pseudovariety $\mathbf{L H}$. For this we will use the results of [2], where Almeida and Azevedo study semidirect products of the form $\mathbf{V} * \mathbf{D}_{k}$, with $\mathbf{V}$ a pseudovariety of semigroups which is not locally trivial and $\mathbf{D}_{k}$ the pseudovariety of all finite semigroups that verify the identity $y x_{1} \cdots x_{k}=x_{1} \cdots x_{k}$. Recall that $\bigcup_{k \geq 1} \mathbf{D}_{k}=\mathbf{D}$. We are interested only in the cases where $\mathbf{V}$ is $\mathbf{H}$, since $\mathbf{L H}=\mathbf{H} * \mathbf{D}=$ $\bigcup_{k \geq 1} \mathbf{H} * \mathbf{D}_{k}$, and so we only recall the corresponding results.

Denote by $L_{k}=A^{\leq k}$ the (factorial) language of all words over $A$ of length at most $k$ and notice that $\ddot{L}_{k}=A^{k+1}$. For a word $u \in A^{+}$, let $\dot{i}_{k}(u)\left(\right.$ resp. $\left.\mathrm{t}_{k}(u)\right)$ be the longest prefix (resp. suffix) of $u$ of length at most $k$. A function $\hbar: A^{+} \rightarrow G$ into a group $G$ is said to be a $k$-superposition homomorphism if $\hbar(u v)=\hbar(u) \hbar\left(\mathrm{t}_{k}(u) v\right)=\hbar\left(u \mathrm{i}_{k}(v)\right) \hbar(v)$ and $\hbar(w)=1_{G}$ for every $u, v \in A^{+}$and $w \in L_{k}$. Notice that, for a word $w \in A^{+} \backslash L_{k}$, $\hbar(w)=\hbar\left(w_{1}\right) \hbar\left(w_{2}\right) \cdots \hbar\left(w_{|w|-k}\right)$ where $w_{1}, w_{2}, \ldots, w_{|w|-k} \in \ddot{L}_{k}$ are the successive factors of length $k+1$ of $w$. Therefore a $k$-superposition homomorphism $\hbar: A^{+} \rightarrow G$ is defined by $\hbar\left(\ddot{L}_{k}\right)$.

Given a $k$-superposition homomorphism $\hbar: A^{+} \rightarrow G$ into a group $G \in \mathbf{H}$, let

$$
M_{k}(G, \hbar)=\left\{\left(v, 1_{G}, v\right) \mid v \in L_{k-1}\right\} \cup\left(A^{k} \times G \times A^{k}\right)
$$

be the semigroup with multiplication given, for every $u, v, u^{\prime}, v^{\prime} \in L_{k}$ and $g, g^{\prime} \in G$, by

$$
(u, g, v)\left(u^{\prime}, g^{\prime}, v^{\prime}\right)=\left(\dot{i}_{k}\left(u u^{\prime}\right), g \hbar\left(v u^{\prime}\right) g^{\prime}, \mathrm{t}_{k}\left(v v^{\prime}\right)\right) .
$$

Note that $I=A^{k} \times G \times A^{k}$ is the minimal ideal of $M_{k}(G, \hbar)$, precisely the Rees matrix semigroup $\mathcal{M}\left[G ; A^{k}, A^{k} ; P\right]$ with $P=(\hbar(u v))_{u, v \in A^{k}}$, and that all the elements of $\left\{\left(v, 1_{G}, v\right) \mid\right.$ $\left.v \in L_{k-1}\right\}$ are non-regular, whence $M_{k}(G, \hbar) \in \mathbf{L H}$. Furthermore, we have the following specialization of [1, Corollary 10.6.8].

Proposition 3.1 The pseudovariety $\mathbf{L H}$ is generated by the semigroups of the form $M_{k}(G, \hbar)$ with $k \geq 1, G \in \mathbf{H}, A$ an alphabet and $\hbar: A^{+} \rightarrow G$ a k-superposition homomorphism.

Given a semigroup $M_{k}(G, \hbar)$, we now identify a finite semigroup $\mathcal{S}[G, L, \mathrm{f}]$, also denoted $S_{k}(G, \mathrm{f})$, such that $M_{k}(G, \hbar)$ is a subsemigroup of a homomorphic image of $S_{k}(G, \mathrm{f})$. The group $G$ is of course the same for the two semigroups. We take for $L$ the language $L_{k}=A^{\leq k}$. This way the minimal ideal $I=A^{k} \times G \times A^{k}$ of $M_{k}(G, \hbar)$ is a subset of the minimal ideal $J=L_{k}^{1} \times G \times L_{k}^{1}$ of $S_{k}(G, \mathrm{f})$. Finally, notice that $\hbar$ has domain $A^{+}$whereas we need to define a function $\mathrm{f}$ with domain $L_{k} \cup \ddot{L}_{k}\left(=L_{k+1}\right)$ and we want to make $I$ a subsemigroup of $J$. But this is no problem since $\hbar$ is a $k$-superposition homomorphism and so $\hbar\left(L_{k}\right)=\left\{1_{G}\right\}$ and the value of $\hbar$ on $A^{+} \backslash L_{k}$ is determined by its value on $\ddot{L}_{k}$. We let $\mathrm{f}$ be the restriction of $\hbar$ to $L_{k} \cup \ddot{L}_{k}$. Therefore, with the above choices, $\hbar$ is precisely the restriction of $\hat{\mathrm{f}}$ to $A^{+}$. Since the function $\hat{\mathrm{f}}$ is the one that determines the structure matrix of the minimal ideal $J$ of $S_{k}(G, \mathrm{f})$, 
the objective of turning $I$ into a subsemigroup of $J$ is guaranteed. The difference between $M_{k}(G, \hbar)$ and $S_{k}(G, \mathrm{f})$ is not only in their minimal ideals: while the set of non-regular elements of $S_{k}(G, \mathrm{f})$ is $L_{k}$, the set of non-regular elements of $M_{k}(G, \hbar)$ is in bijection with $L_{k-1}$. That is, each word $u \in A^{k}$ is an element of $S_{k}(G, \mathrm{f})$ that lies $\mathcal{J}$-above the minimal ideal while in $M_{k}(G, \hbar)$ it corresponds to the element $\left(u, 1_{G}, u\right)$ that lies in the minimal ideal. On the other hand $S_{k}(G, \mathrm{f})$ also has an element $\left(u, 1_{G}, u\right)$. So, we construct a new semigroup in which the elements $u$ and $\left(u, 1_{G}, u\right)$ are identified (and only these ones).

Lemma 3.2 With the above choices for $L$ and $\mathrm{f}$, let $S_{k}^{\prime}(G, \mathrm{f})$ be a semigroup defined by the presentation $\left\langle X \mid R^{\prime}\right\rangle$, where $R^{\prime}=R_{G} \cup R_{\mathrm{f}} \cup\left\{u=u e u: u \in A^{k}\right\}$.

(a) The semigroup $S_{k}^{\prime}(G, \mathrm{f})$ is a homomorphic image of $S_{k}(G, \mathrm{f})$.

(b) $\rho_{R^{\prime}}=\rho_{R} \cup\left\{u=\right.$ ueu $\left.: u \in A^{k}\right\}$, where $R=R_{G} \cup R_{\mathrm{f}}$.

Proof. To deduce $(a)$ it suffices to note that $S_{k}^{\prime}(G, \mathrm{f})$ is defined by a presentation that differs from the one defining $S_{k}(G, \mathrm{f})$ only in having the extra relations $u=u e u\left(u \in A^{k}\right)$.

The inclusion $\rho_{R} \cup\left\{u=u e u: u \in A^{k}\right\} \subseteq \rho_{R^{\prime}}$ is immediate from the definition of a congruence generated by a relation. To prove the reverse inclusion, let $w, z \in X^{+}$and suppose that $(w=z) \in \rho_{R^{\prime}}$ and $(w=z) \notin\left\{u=u e u: u \in A^{k}\right\}$. We need to prove that $(w=z) \in \rho_{R}$. This holds trivially if the relation $w=z$ is deduced without using the relations $u=u e u$ $\left(u \in A^{k}\right)$. We assume, without loss of generality, that $w=w^{\prime} u w^{\prime \prime}$ and $z=w^{\prime} u e u w^{\prime \prime}$ with $u \in A^{k}$ and $w^{\prime}$ or $w^{\prime \prime}$ non-empty. We treat only the case in which $w^{\prime}$ is non-empty since the case $w^{\prime \prime}$ non-empty is similar. If $\mathrm{t}_{1}\left(w^{\prime}\right) \in A_{G}$ then, by (2.4) and using relation $r_{u}$, one deduces that $z=w^{\prime}$ eueuw" $=w^{\prime} e u w^{\prime \prime}=w$ in $X^{+} / \rho_{R}$. If $\mathrm{t}_{1}\left(w^{\prime}\right)=a \in A$ then $w^{\prime}=w^{\prime \prime \prime} a$ for some $w^{\prime \prime \prime} \in X^{*}$ and $a u \in A^{k+1}=\ddot{L}_{k}$. Notice that $(a u)_{\alpha}=i_{k}(a u)$ and $(a u)_{\omega}=\mathrm{t}_{k}(a u)=u$. Hence, using relation $r_{a u}$ one deduces that $z=w^{\prime \prime \prime} i_{k}(a u) \mathrm{f}(a u) u e u w^{\prime \prime}$ is in $\rho_{R}$. Since $\mathrm{f}(a u) \in A_{G}^{+}$ we may now proceed as in the previous case. So, using relations $r_{u}$ and $r_{a u}$, we deduce that $z=w^{\prime \prime \prime} \mathbf{i}_{k}(a u) f(a u) u w^{\prime \prime}=w^{\prime \prime \prime} a u w^{\prime \prime}=w$ in $X^{+} / \rho_{R}$. Therefore, in both cases, $(w=z) \in \rho_{R}$. This proves $(b)$.

Note that, informally speaking, Lemma $3.2(b)$ states that the relation $u=u e u\left(u \in A^{k}\right)$ makes no identifications on $S_{k}(G, \mathrm{f})$ other than the elements $u$ and $\left(u, 1_{G}, u\right)$. It should now be clear that the mapping $\phi: M_{k}(G, \hbar) \rightarrow S_{k}^{\prime}(G, \mathrm{f})$ given, for $v \in L_{k-1}, u_{1}, u_{2} \in A^{k}$ and $g \in G$, by $\phi(v, 1, v)=v$ and $\phi\left(u_{1}, g, u_{2}\right)=\left(u_{1}, g, u_{2}\right)$ is a monomorphism from $M_{k}(G, \hbar)$ into $S_{k}^{\prime}(G, \mathrm{f})$. The following result is then an immediate consequence of Proposition 3.1 and Lemma 3.2 .

Corollary 3.3 The pseudovariety $\mathbf{L H}$ is generated by the semigroups of the form $S_{k}(G, \mathrm{f})$ with $k \geq 1, G \in \mathbf{H}, A$ an alphabet and $\mathrm{f}: L_{k+1} \rightarrow G$ with $\mathrm{f}\left(L_{k}\right)=\left\{1_{G}\right\}$.

We observe that, in general, the semigroup $M_{k}(G, \hbar)$ is not a homomorphic image of $S_{k}(G, \mathrm{f})$. That is, it is not possible to obtain a presentation for $M_{k}(G, \hbar)$ simply by adding 
new relations to the presentation that defines $S_{k}(G, \mathrm{f})$. For instance, consider the alphabet $A=\{a\}$, the cyclic group $G=\left\langle g \mid g^{3}=g\right\rangle=\{g, e\}$ of order $2, k=1$ and $\hbar\left(a^{2}\right)=g$. Then $M_{k}(G, \hbar)=\{a\} \times G \times\{a\} \cong G$ and $S_{k}(G, \mathrm{f})=\left\langle a, g \mid g^{3}=g, e a e=e, a^{2}=a g a\right\rangle=$ $\{a\} \cup(\{1, a\} \times G \times\{1, a\})$. In order to obtain $M_{k}(G, \hbar)$ as a homomorphic image of $S_{k}(G, f)$ one should identify the idempotents $(1, e, 1)$ and $(a, g, a)$ of $S_{k}(G, f)$, by adding the relation $e=a g a$ to the above presentation. But then we would have $a^{2}=e$ and so $e=e e=a^{2} a^{2}=$ $a a^{2} a=a e a=a^{2} g a^{2}=e g e=g$ in the resulting semigroup. This semigroup would therefore be aperiodic and so different from $M_{k}(G, \hbar)$.

\section{Canonical forms for $\kappa$-terms of rank 1}

A $\kappa$-term is a formal expression obtained from letters of an alphabet $A$ using two operations: the binary concatenation and the unary $(\omega-1)$-power. The rank of a $\kappa$-term is the maximum number of nested $(\omega-1)$-powers in it. A $\kappa$-term has a natural interpretation on each finite semigroup $S$ : the concatenation is viewed as the semigroup multiplication while the $(\omega-1)$ power is interpreted as the unary operation which sends each element $s$ of $\mathrm{S}$ to the inverse of $s^{\omega+1}\left(=s s^{\omega}\right)$ in the maximal subgroup containing the unique idempotent power $s^{\omega}$ of $s$. For a class $\mathcal{C}$ of finite semigroups and $\kappa$-terms $\pi_{1}$ and $\pi_{2}$, we say that $\mathcal{C}$ satisfies the $\kappa$-identity $\pi_{1}=\pi_{2}$, and write $\mathcal{C} \models \pi_{1}=\pi_{2}$, if $\pi_{1}$ and $\pi_{2}$ have the same interpretation over every semigroup of $\mathcal{C}$. The $\kappa$-word problem for $\mathcal{C}$ consists in deciding, given a $\kappa$-identity $\pi_{1}=\pi_{2}$, whether $\mathcal{C} \models \pi_{1}=\pi_{2}$. A solution for this problem has been obtained for some important pseudovarieties and we will soon present solutions for the pseudovarieties $\mathbf{S}$ [5] and LG [7]. In the current paper we will treat an instance of the problem by showing that $\mathbf{S}$ and $\mathbf{L G}$ satisfy the same $\kappa$-identities $\pi=\rho$ where $\pi$ and $\rho$ have rank at most 1 , and that it is decidable whether $\mathbf{S}$ and $\mathbf{L G}$ satisfy $\pi=\rho$. For that, we first reduce each $\kappa$-term of rank 1 to a certain canonical form, similar to the normal form introduced by McCammond in [12] to solve the $\omega$-word problem for the pseudovariety $\mathbf{A}$ of finite aperiodic semigroups. The definition of an $\omega$-term differs from that of a $\kappa$-term only in the use of the $\omega$-power instead of the $(\omega-1)$ power (we remark that the two operations coincide on the pseudovariety $\mathbf{A}$ ). Then we use our test-semigroups $\mathcal{S}(G, L, \mathrm{f})$ to separate two distinct canonical forms.

A word is said to be primitive if it cannot be written in the form $u^{n}$ with $n>1$. We say that two words $w$ and $z$ are conjugate if there exist words $u, v \in A^{*}$ such that $w=u v$ and $z=v u$. Let an order be fixed for the letters of the alphabet $A$. A Lyndon word is a primitive word which is minimal, with respect to the lexicographic ordering, in its conjugacy class. For combinatorial properties involving Lyndon words that are relevant for the remaining of this paper, the reader is referred to [3], where an alternative proof of correctness of McCammond's normal form algorithm over $\mathbf{A}$ is presented.

We employ the following notation for $\kappa$-terms, where $n>0: x^{\omega+n}$ represents $x^{\omega} x^{n} ; x^{\omega+0}$ is $x^{\omega} ; x^{\omega-n}$ denotes $\left(x^{\omega-1}\right)^{n}$. The $\kappa$-terms of rank 0 are the words from $A^{+}$and they are all 
considered to be in canonical form. A $\kappa$-term of rank 1 is an expression $\pi$ of the form

$$
\pi=u_{0} x_{1}^{\omega+q_{1}} u_{1} x_{2}^{\omega+q_{2}} \cdots x_{m}^{\omega+q_{m}} u_{m}
$$

with $m \geq 1, u_{0}, \ldots, u_{m} \in A^{*}, x_{1}, \ldots, x_{m} \in A^{+}$and $q_{1}, \ldots, q_{m} \in \mathbb{Z}$. Using the terminology of McCammond [12], each factor of the form $x_{i}^{\omega+q_{i}} u_{i} x_{i+1}^{\omega+q_{i+1}}$ will be called a crucial portion of $\pi$. The prefix $u_{0} x_{1}^{\omega+q_{1}}$ and the suffix $x_{m}^{\omega+q_{m}} u_{m}$ will be called respectively the initial portion and the final portion of $\pi$.

Definition 4.1 (Canonical form in rank 1) The $\kappa$-term $\pi$ in (4.1) is in canonical form if

(a) each $x_{i}$ is a Lyndon word;

(b) $x_{i}$ is not a suffix of $u_{i-1}$ for $1 \leq i \leq m$;

(c) $x_{i}$ is not a prefix of $u_{i} x_{i+1}^{\left|x_{i}\right|}$ for $1 \leq i \leq m$, where $x_{m+1}$ is the empty word.

For instance, let $a, b \in A$ be letters such that $a<b$. The $\kappa$-terms $b(a b b)^{\omega-1} b^{\omega+2}$ and $a^{\omega+3} b(a a b)^{\omega} a a(a b)^{\omega-2}$ are in canonical form, as is also any $\kappa$-term of the type $\left(a x^{n}\right)^{\omega+p} x^{\omega+q}$ or $y^{\omega+p} b\left(y^{n} b\right)^{\omega+q}$ where $x \in\{a, b\}^{*} b\{a, b\}^{*}$ and $y \in\{a, b\}^{*} a\{a, b\}^{*}$ are Lyndon words and $n, p, q \in \mathbb{Z}$ with $n \geq 1$. On the contrary, the $\kappa$-terms $a(b a b)^{\omega-1}(a a)^{\omega}$ and $(a b)^{\omega} a b a b^{\omega-1} b^{3}$ are not in canonical form. We remark that our condition to the canonical form of each crucial portion is different from the one that McCammond [12] imposed to crucial portions of $\omega$ terms. That is, if $x_{i}^{\omega+q_{i}} u_{i} x_{i+1}^{\omega+q_{i+1}}$ is in canonical form, then the $\omega$-term $x_{i}^{\omega} u_{i} x_{i+1}^{\omega}$ may not be in McCammond's normal form. To each crucial portion $x_{i}^{\omega+q_{i}} u_{i} x_{i+1}^{\omega+q_{i+1}}$ there is an associated bi-infinite word $\cdots x_{i} x_{i} u_{i} x_{i+1} x_{i+1} \cdots$, which we denote by $x_{i}^{-\infty} u_{i} x_{i+1}^{+\infty}$. From the definition of canonical form, it is easy to deduce that, for $i, j \in\{1, \ldots, m-1\}$, the bi-infinite words $x_{i}^{-\infty} u_{i} x_{i+1}^{+\infty}$ and $x_{j}^{-\infty} u_{j} x_{j+1}^{+\infty}$ coincide if and only if $x_{i}=x_{j}, u_{i}=u_{j}$ and $x_{i+1}=x_{j+1}$.

As we shall see below, for each $\kappa$-term $\alpha$ of rank 1 there is a $\kappa$-term $\alpha^{\prime}$ in canonical form such that $\mathbf{S} \models \alpha=\alpha^{\prime}$. The $\kappa$-term $\alpha^{\prime}$ is unique by Theorem 5.1 below and so we call it the canonical form of $\alpha$. Moreover it can be computed from $\alpha$ by applying elementary changes resulting from reading in either direction the following $\kappa$-identities, where $i, j, n \in \mathbb{Z}$ with $n>0$,
1. $\left(x^{n}\right)^{\omega+j}=x^{\omega+n j}$
$3_{R} \cdot x^{\omega+i} x=x^{\omega+i+1}$
2. $x^{\omega+i} x^{\omega+j}=x^{\omega+i+j}$
$3_{L} \cdot x x^{\omega+i}=x^{\omega+i+1}$
4. $(x y)^{\omega+i} x=x(y x)^{\omega+i}$

These $\kappa$-identities are easily shown to be valid in all finite semigroups and so $\alpha=\alpha^{\prime}$ is indeed verified by $\mathbf{S}$. If a subterm given by the left side of a $\kappa$-identity of type 1-3 is replaced in a $\kappa$-term by the right side of the $\kappa$-identity, then we say there is a contraction of that type. If the replacement is done in the opposite direction then we say that there is an expansion of that type. An application of the $\kappa$-identity 4 , in either direction, will be called a shift. For 
example, consider the $\kappa$-term $\alpha=(b a b a b a)^{\omega} b^{\omega-3} b(b b)^{\omega+1}$. The following sequence of $\kappa$-terms, starting in $\alpha$, is derived from the $\kappa$-identities $1-4$ above,

$$
\begin{aligned}
\alpha & =(b a)^{\omega} b^{\omega-3} b(b b)^{\omega+1}=(b a)^{\omega} b^{\omega-3} b b^{\omega+2}=(b a)^{\omega-1} b a b^{\omega-3} b b^{\omega+2} \\
& =b(a b)^{\omega-1} a b^{\omega-3} b b^{\omega+2}=b(a b)^{\omega-1} a b^{\omega-2} b^{\omega+2}=b(a b)^{\omega-1} a b^{\omega} \\
& =b(a b)^{\omega-1} a b b^{\omega-1}=b(a b)^{\omega} b^{\omega-1} .
\end{aligned}
$$

The two first steps in this derivation are contractions of type 1, the third step is an expansion of type $3_{R}$, the fourth is a shift, the fifth is a contraction of type $3_{R}$, the sixth step is a contraction of type 2 , the seventh is an expansion of type $3_{L}$ and the final step is a contraction of type $3_{R}$. As $b(a b)^{\omega} b^{\omega-1}$ is in canonical form, we conclude that this $\kappa$-term is the canonical form of $\alpha$. The steps of the algorithm to compute the canonical form of an arbitrary $\kappa$-term of rank 1 may be described as follows.

(1) Apply all possible contractions of type 1.

(2) By means of an expansion of type 3 and a shift, write each infinite power in the form $x^{\omega+q}$ where $x$ is a Lyndon word.

(3) Apply all possible contractions of type 3 .

(4) Apply all possible contractions of type 2 .

(5) Standardize each crucial portion $x^{\omega+p} u y^{\omega+q}$ as follows. By step (3), $x$ is not a prefix and $y$ is not a suffix of $u$. Let $\ell$ be the minimum non negative integer such that $\left|u y^{\ell}\right| \geq|x|$. If $x$ is not a prefix of $u y^{\ell}$ then the crucial portion $x^{\omega+p} u y^{\omega+q}$ is already in canonical form. Otherwise $\ell \neq 0$. In this case, apply $\ell$ expansions of type $3_{L}$ to the limit term on the right side of the crucial portion, followed by all $n \geq 1$ possible contractions of type $3_{R}$. As shown in [5], the crucial portion $x^{\omega+p+n} v y^{\omega+q-\ell}$ thus obtained is already in canonical form.

It is not difficult to check that the above procedure does indeed transform any $\kappa$-term of rank 1 into one in canonical form.

\section{$5 \quad$ Identities involving $\kappa$-terms of rank at most 1}

The main objective of this section is to prove that $\mathbf{S}$ and $\mathbf{L G}$ satisfy the same $\kappa$-identities involving $\kappa$-terms of rank at most 1 . This property cannot, obviously, be extended to rank 2 since $\mathbf{S}$ does not verify the $\kappa$-identity $\left(x^{\omega} y x^{\omega}\right)^{\omega}=x^{\omega}$ while $\mathbf{L G}$ verifies it (in fact this $\kappa$-identity defines $\mathbf{L G})$.

Consider rank $1 \kappa$-terms $\pi=u_{0}^{\prime} x_{1}^{\omega+q_{1}} u_{1} \cdots x_{m}^{\omega+q_{m}} u_{m}$ and $\rho=u_{m}^{\prime} x_{m+1}^{\omega+q_{m+1}} u_{m+1} \cdots x_{n}^{\omega+q_{n}} u_{n}$ in canonical form. We associate to the $\kappa$-identity $\pi=\rho$ an identity $\mathrm{w}_{\pi}=\mathrm{w}_{\rho}$ and a finite local group $S_{\pi, \rho}$, of the form $\mathcal{S}(G, L, \mathrm{f})$, as follows. As we shall see, the identity $\mathrm{w}_{\pi}=\mathrm{w}_{\rho}$ (together with another condition easy to verify) serves to decide whether the $\kappa$-identity $\pi=\rho$ holds over 
LG and $S_{\pi, \rho}$ is a test-semigroup for $\pi=\rho$. We set $I_{n}=\{1, \ldots, n\}$ and $I_{m, n}=I_{n} \backslash\{m, n\}$. We also set, for each $i \in I_{n}, \ell_{i}=\left|x_{i}\right|$ and $x_{i}=a_{i 1} a_{i 2} \cdots a_{i \ell_{i}}$ with $a_{i 1}, a_{i 2}, \ldots, a_{i \ell_{i}} \in A$. For each $j \in\left\{1, \ldots, \ell_{i}\right\}$, we let $x_{i j}$ be the conjugate $x_{i j}=a_{i j} \cdots a_{i \ell_{i}} a_{i 1} \cdots a_{i j-1}$ of $x_{i}$ and notice that $x_{i 1}=x_{i}$. Now, let $\mathbb{q}=1+\max \left\{\left|q_{i}\right|: i \in I_{n}\right\}$ and, for each $i \in I_{n}$, consider the positive integer $\mathbb{q}_{i}=\mathbb{q}+q_{i}$. We associate a variable $\mathrm{v}_{x}$ to each word $x \in A^{+}$and a variable $\mathrm{v}_{x, u, y}$ to each triple $(x, u, y)$ of words of $A^{+}$. Let $\mathrm{V}=\left\{\mathrm{v}_{x_{i}}, \mathrm{v}_{x_{j}, u_{j}, x_{j+1}}: i \in I_{n}, j \in I_{m, n}\right\}$. We associate to $\pi$ and $\rho$ the following words $\mathrm{w}_{\pi}, \mathrm{w}_{\rho} \in \mathrm{V}^{+}$,

$$
\begin{aligned}
& \mathbf{w}_{\pi}=\mathbf{u}_{0}^{\prime} \mathbf{v}_{x_{1}}^{q_{1}} \mathbf{v}_{x_{1}, u_{1}, x_{2}} \mathbf{v}_{x_{2}}^{q_{2}} \cdots \mathbf{v}_{x_{m-1}, u_{m-1}, x_{m}} \mathbf{v}_{x_{m}}^{q_{m}} \mathbf{u}_{m}, \\
& \mathbf{w}_{\rho}=\mathbf{u}_{m}^{\prime} \mathbf{v}_{x_{m+1}}^{q_{m+1}} \mathbf{v}_{x_{m+1}, u_{m+1}, x_{m+2}} \mathbf{v}_{x_{m+2}}^{q_{m+2}} \cdots \mathbf{v}_{x_{n-1}, u_{n-1}, x_{n}} \mathbf{v}_{x_{n}}^{q_{n}} \mathbf{u}_{n},
\end{aligned}
$$

where $\mathbf{u}_{0}^{\prime}\left(\right.$ resp. $\left.\mathbf{u}_{m}, \mathbf{u}_{m}^{\prime}, \mathbf{u}_{n}\right)$ is the variable $\mathbf{v}_{x_{1}}$ (resp. $\left.\mathbf{v}_{x_{m}}, \mathbf{v}_{x_{m+1}}, \mathbf{v}_{x_{n}}\right)$ if $u_{0}^{\prime}$ (resp. $u_{m}, u_{m}^{\prime}, u_{n}$ ) is non-empty and is the empty word otherwise. This completes the definition of the identity $\mathrm{w}_{\pi}=\mathrm{w}_{\rho}$.

Let us now define the test-semigroup $S_{\pi, \rho}=\mathcal{S}(G, L, \mathrm{f})$. Denote by $F_{\vee}$ the free group generated by the alphabet $\mathrm{V}$. We select first a group homomorphism $\eta: F_{\mathrm{V}} \rightarrow G$ into a finite group $G$, associating to each variable $\mathrm{v}_{*}$ an element $\eta\left(\mathrm{v}_{*}\right)=g_{*}$, in such a way that: if the identity $\mathrm{w}_{\pi}=\mathrm{w}_{\rho}$ is non-trivial, then $g_{\pi}=\eta\left(\mathrm{w}_{\pi}\right)$ and $g_{\rho}=\eta\left(\mathrm{w}_{\rho}\right)$ are distinct elements of $G$. We observe the following

Claim 1 We may assume that each element of $\eta(\mathrm{V})$ has an order at least $2 q$.

Proof. In order to attest the claim, let $C_{2 q}=\langle s\rangle$ denote the cyclic group of order $2 \llbracket$ generated by an element $s$ and consider the group $G^{\prime}=G \times C_{2 \mathrm{q}}$. The elements $g_{*}^{\prime}=\left(g_{*}, s\right)$ of $G^{\prime}$ have an order at least $2 \llbracket$. Let $\eta^{\prime}: F_{\bigvee} \rightarrow G^{\prime}$ be the group homomorphism defined by $\eta^{\prime}\left(\mathrm{v}_{*}\right)=g_{*}^{\prime}$. The first components of $g_{\pi}^{\prime}=\eta^{\prime}\left(\mathrm{w}_{\pi}\right)$ and $g_{\rho}^{\prime}=\eta^{\prime}\left(\mathrm{w}_{\rho}\right)$ are respectively $g_{\pi}$ and $g_{\rho}$. If these are distinct elements of $G$, then $g_{\pi}^{\prime}$ and $g_{\rho}^{\prime}$ are distinct elements of $G^{\prime}$. Therefore, if necessary, we replace $\eta$ by $\eta^{\prime}$ thus proving the claim.

We describe next the language $L$. We will fix three positive integers $i<j<\mathbb{k}$ and define sets of words $W_{\mathrm{i}}, W_{\mathrm{j}}$ and $W_{\mathrm{k}}$ as follows. We choose first an integer $\stackrel{\AA}{\mathrm{s}}>\left|u_{0}^{\prime} x_{1} u_{1} \cdots x_{m} u_{m}\right|+$ $\left|u_{m}^{\prime} x_{m+1} u_{m+1} \cdots x_{n} u_{n}\right|$ and let $W_{\text {i }}=\left\{u_{0}^{\prime} x_{1}^{i}, u_{m}^{\prime} x_{m+1}^{i}, x_{m}^{i} u_{m}, x_{n}^{i} u_{n}\right\}$. Suppose now that $n>$ 2. Each crucial portion $x_{i}^{\omega+q_{i}} u_{i} x_{i+1}^{\omega+q_{i+1}}\left(i \in I_{m, n}\right)$ of $\pi$ or $\rho$ determines a bi-infinite word $x_{i}^{-\infty} u_{i} x_{i+1}^{+\infty}$, that is not periodic (i.e., it is not of the form $\cdots v v v \cdots$, the infinite repetition for both sides of the same finite word $v$ ) by definition of canonical form. Therefore, for every $i, i^{\prime} \in I_{m, n}$ and $§$ large enough, either $x_{i}^{j} u_{i} x_{i+1}^{j}$ does not occur in $x_{i^{\prime}}^{-\infty} u_{i^{\prime}} x_{i^{\prime}+1}^{+\infty}$, or, as a consequence of the rank 1 canonical form definition, it has exactly one occurrence in $x_{i^{\prime}}^{-\infty} u_{i^{\prime}} x_{i^{\prime}+1}^{+\infty}$ (in which case $x_{i}=x_{i^{\prime}}, u_{i}=u_{i^{\prime}}$ and $x_{i+1}=x_{i^{\prime}+1}$ ). We then say that $x_{i}^{j} u_{i} x_{i+1}^{j}$ is synchronized in $x_{i^{\prime}}^{-\infty} u_{i^{\prime}} x_{i^{\prime}+1}^{+\infty}$. We fix one such $₫$ with $₫>\AA$, let $W_{j}=\left\{x_{i}^{j} u_{i} x_{i+1}^{j}: i \in I_{m, n}\right\}$ and observe that $W_{\mathrm{i}}$ and $W_{\mathrm{j}}$ are disjoint sets. If $n=2$ then $\pi$ and $\rho$ have no crucial portions and we let $\AA>\AA$ and take for $W_{j}$ the empty set. For any $n$, we fix at last an integer $\mathbb{k}>\AA$ such that $x_{i q}^{\mathrm{k}} \notin F\left(W_{j}\right)$ for every $i \in I_{n}$ and $q \in\left\{1, \ldots, \ell_{i}\right\}$. Since we may take for $\mathbb{k}$ any sufficiently 
large positive integer, we pick out an option such that $\mathbb{k}^{\prime}=\mathbb{k}+1+\mathbb{q}$ is a multiple of the order of each element $g_{x_{i}}$ with $i \in I_{n}$. Finally, we set $W_{\mathrm{k}}=\left\{x_{i q}^{\mathrm{k}}: i \in I_{n}, q \in\left\{1, \ldots, \ell_{i}\right\}\right\}$ and $L=F\left(W_{\mathrm{i}} \cup W_{\mathrm{j}} \cup W_{\mathrm{k}}\right)$.

We conclude the definition of the semigroup $S_{\pi, \rho}$ by identifying the mapping f : $L \cup \ddot{L} \rightarrow G$. We consider the subset $W_{\mathrm{k}}^{\prime}=\left\{x_{1}^{\mathrm{k}}, \ldots, x_{n}^{\mathrm{k}}\right\}$ of $W_{\mathrm{k}}$ and define

$$
\mathrm{f}(w)= \begin{cases}1_{G} & \text { if } w \in(L \cup \ddot{L}) \backslash\left(W_{j} \cup W_{\mathrm{k}}^{\prime}\right) \\ g_{x_{i}}^{-1} g_{x_{i}, u_{i}, x_{i+1}} g_{x_{i+1}}^{-t_{i}-1} & \text { if } w=x_{i}^{\mathrm{j}} u_{i} x_{i+1}^{\mathrm{j}} \in W_{j} \\ g_{x_{i}} & \text { if } w=x_{i}^{\mathrm{k}} \in W_{\mathrm{k}}^{\prime}\end{cases}
$$

where $t_{i} \geq 0$ is the biggest integer such that $x_{i+1}^{t_{i}}$ is a suffix of $x_{i}^{j} u_{i}$.

We may now prove the main result of this section.

Theorem 5.1 Under the above assumptions and with the above notations, the following conditions are equivalent:

(a) $\mathbf{S} \models \pi=\rho$.

(b) $\mathbf{L G} \models \pi=\rho$.

(c) $S_{\pi, \rho} \models \pi=\rho$.

(d) $\mathrm{w}_{\pi}=\mathrm{w}_{\rho}, u_{0}^{\prime}=u_{m}^{\prime}$ and $u_{m}=u_{n}$.

(e) $\pi$ and $\rho$ are the same $\kappa$-term.

Proof. The sequence of implications $(d) \Rightarrow(e) \Rightarrow(a) \Rightarrow(b) \Rightarrow(c)$ holds trivially. It remains therefore to prove the implication $\left[(c) \Leftrightarrow(d)\right.$. Thus suppose that $S_{\pi, \rho} \models \pi=\rho$ and let $\phi$ : $T_{A}^{\kappa} \rightarrow S_{\pi, \rho}$ be the homomorphism of $\kappa$-semigroups that coincides with $\varphi \circ \check{f}$ on $A^{+}$, where $T_{A}^{\kappa}$ denotes the $\kappa$-semigroup of all $\kappa$-terms and $\varphi$ is the isomorphism from $\mathcal{Z}[G, L, \mathrm{f}]$ onto $S_{\pi, \rho}$ defined in Section 2, Notice that, $\phi(w)=w$ when $w \in L$ and, for $w \in A^{+} \backslash L$ with $\operatorname{sc}_{L}[w]=\left(w_{0}, \ddot{w}_{1}, w_{1}, \ldots, \ddot{w}_{p}, w_{p}\right), \check{\mathrm{f}}(w)=w_{0} \overline{\mathrm{f}}(w) w_{p}$ and $\phi(w)=\left(w_{0}, \overline{\mathrm{f}}(w), w_{p}\right)$ where $\overline{\mathrm{f}}(w)=$ $\mathrm{f}\left(\ddot{w}_{1}\right) \mathrm{f}\left(w_{1}\right) \mathrm{f}\left(\ddot{w}_{2}\right) \cdots \mathfrak{f}\left(\ddot{w}_{p}\right)$. We begin by computing the value under $\phi$ of each power $x_{i}^{\omega+q_{i}}$ with $i \in I_{n}$. By definition of $W_{\mathrm{k}}, x_{i}^{\mathrm{k}}$ belongs to $L$, so that $\phi\left(x_{i}^{\mathrm{k}}\right)=x_{i}^{\mathrm{k}}$. On the other hand, by the assumption on $\mathbb{k}$, it is clear that $x_{i}^{\mathrm{k}+1} \notin L$, whence $\phi\left(x_{i}^{\mathrm{k}+1}\right)$ belongs to the minimal ideal of $S_{\pi, \rho}$. Therefore, the kernel $K_{x_{i}}$ of the subsemigroup $\left\langle x_{i}\right\rangle$ of $S_{\pi, \rho}$ generated by $x_{i}\left(=\phi\left(x_{i}\right)\right)$, is of the form

$$
K_{x_{i}}=\left\{\phi\left(x_{i}^{\mathrm{k}+1}\right), \phi\left(x_{i}^{\mathrm{k}+2}\right), \ldots, \phi\left(x_{i}^{\mathrm{k}+p_{i}}\right)\right\}
$$

with $p_{i} \geq 1$ minimal such that $\phi\left(x_{i}^{\mathrm{k}+1+p_{i}}\right)=\phi\left(x_{i}^{\mathrm{k}+1}\right)$ and thus

$$
\exists q_{i}^{\prime} \in\left\{1, \ldots, p_{i}\right\}, \phi\left(x_{i}^{\omega+q_{i}}\right)=\phi\left(x_{i}^{\mathrm{k}+q_{i}^{\prime}}\right) .
$$

The positive integers $p_{i}$ and $q_{i}^{\prime}$ are specified in the following lemma.

Lemma $5.2(a) p_{i}$ is the order of the element $g_{x_{i}}$ in $G$; 
(b) $q_{i}^{\prime}=\mathbb{q}_{i}+1$ (which is equivalent to saying that $\left.\mathbb{k}+q_{i}^{\prime}=\mathbb{k}^{\prime}+q_{i}\right)$.

Proof. Notice first that, for every $j \in\left\{1, \ldots, \ell_{i}\right\}$, the word $v_{j}=x_{i j}^{\mathrm{k}} a_{i j}$ belongs to $\ddot{L}$, $\left(v_{j}\right)_{\alpha}=x_{i j}^{\mathrm{k}},\left(v_{j}\right)_{\omega}=x_{i j+1}^{\mathrm{k}}$ if $j \neq \ell_{i}$ and $\left(v_{\ell_{i}}\right)_{\omega}=x_{i 1}^{\mathrm{k}}$. Hence, one verifies that, for $q \geq 1$,

$$
\operatorname{sc}_{L}\left[x_{i}^{\mathrm{k}+q}\right]=\left(x_{i 1}^{\mathrm{k}}, v_{1}, \ldots, x_{i \ell_{i}}^{\mathrm{k}}, v_{\ell_{i}}, x_{i 1}^{\mathrm{k}}, v_{1}, \ldots, x_{i \ell_{i}}^{\mathrm{k}}, v_{\ell_{i}}, \ldots, x_{i 1}^{\mathrm{k}}, v_{1}, \ldots, x_{i \ell_{i}}^{\mathrm{k}}, v_{\ell_{i}}, x_{i 1}^{\mathrm{k}}\right)
$$

with $q$ repetitions of $x_{i 1}^{\mathrm{k}}, v_{1}, \ldots, x_{i \ell_{i}}^{\mathrm{k}}, v_{\ell_{i}}$. Therefore,

$$
\overline{\mathrm{f}}\left(x_{i}^{\mathrm{k}+q}\right)=\mathrm{f}\left(x_{i 1}^{\mathrm{k}}\right)^{-1}\left(\mathrm{f}\left(x_{i 1}^{\mathrm{k}}\right) \mathrm{f}\left(v_{1}\right) \cdots \mathrm{f}\left(x_{i \ell_{i}}^{\mathrm{k}}\right) \mathrm{f}\left(v_{\ell_{i}}\right)\right)^{q} .
$$

On the other hand, $\mathrm{f}\left(x_{i 1}^{\mathrm{k}}\right)=g_{x_{i}}$ and $\mathrm{f}\left(v_{j}\right)=1_{G}$ for all $i$ and $j$, and $\mathrm{f}\left(x_{i j}^{\mathrm{k}}\right)=1_{G}$ for $j \neq 1$. It then follows that

$$
\phi\left(x_{i}^{\mathrm{k}+q}\right)=\left(x_{i}^{\mathrm{k}}, \overline{\mathrm{f}}\left(x_{i}^{\mathrm{k}+q}\right), x_{i}^{\mathrm{k}}\right)=\left(x_{i}^{\mathrm{k}}, g_{x_{i}}^{q-1}, x_{i}^{\mathrm{k}}\right) .
$$

We may now employ (5.2) to deduce that, for $p \geq 1, \phi\left(x_{i}^{\mathrm{k}+1+p}\right)=\phi\left(x_{i}^{\mathrm{k}+1}\right)$ if and only if $g_{x_{i}}^{p}=1_{G}$. Hence, the least positive integer $p_{i}$ such that $\phi\left(x_{i}^{\mathrm{k}+1+p_{i}}\right)=\phi\left(x_{i}^{\mathrm{k}+1}\right)$ is the order of $g_{x_{i}}$, thus proving $(a)$.

For $(b)$, we show that $\mathbb{k}+q_{i}^{\prime}=\mathbb{k}^{\prime}+q_{i}$. In view of Claim $1, p_{i} \geq 2 \mathbb{q}$, whence $\mathbb{k}^{\prime}+q_{i} \in$ $\left\{\mathbb{k}+1, \ldots, \mathbb{k}+p_{i}\right\}$. Therefore, to prove the equality $\mathbb{k}+q_{i}^{\prime}=\mathbb{k}^{\prime}+q_{i}$ it suffices to verify that $\phi\left(x_{i}^{\omega}\right)=\phi\left(x_{i}^{\mathrm{k}^{\prime}}\right)$. For this, using (5.2) and the fact that $g_{x_{i}}^{\mathrm{k}^{\prime}}=1_{G}$, since $p_{i}$ divides $\mathbb{k}^{\prime}$ by the choice of $\mathbb{k}^{\prime}$, we deduce

$$
\phi\left(\left(x_{i}^{\mathrm{k}^{\prime}}\right)^{2}\right)=\phi\left(x_{i}^{\mathrm{k}+1+\mathrm{q}+\mathrm{k}^{\prime}}\right)=\left(x_{i}^{\mathrm{k}}, g_{x_{i}}^{\mathrm{q}+\mathrm{k}^{\prime}}, x_{i}^{\mathrm{k}}\right)=\left(x_{i}^{\mathrm{k}}, g_{x_{i}}^{\mathrm{q}}, x_{i}^{\mathrm{k}}\right)=\phi\left(x_{i}^{\mathrm{k}^{\prime}}\right) .
$$

Thus, $\phi\left(x_{i}^{\mathrm{k}^{\prime}}\right)$ is an idempotent power of $\phi\left(x_{i}\right)$, whence $\phi\left(x_{i}^{\omega}\right)=\phi\left(x_{i}^{\mathrm{k}^{\prime}}\right)$.

Using (5.1), (5.2), Lemma $5.2(b)$ and the fact that $\phi$ and $\check{f}$ are homomorphisms such that $\check{\mathrm{f}} \circ \check{\mathrm{f}}=\check{\mathrm{f}}$, one deduces that

$$
\begin{aligned}
\phi(\pi) & =\phi\left(u_{0}^{\prime} x_{1}^{\mathrm{k}+\mathrm{q}_{1}+1} u_{1} x_{2}^{\mathrm{k}+\mathrm{q}_{2}+1} \cdots u_{m-1} x_{m}^{\mathrm{k}+\mathrm{q}_{m}+1} u_{m}\right) \\
& =\varphi\left(\tilde{\mathrm{f}}\left(u_{0}^{\prime} x_{1}^{\mathrm{k}} g_{x_{1}}^{\mathrm{q}_{1}} x_{1}^{\mathrm{k}} u_{1} x_{2}^{\mathrm{k}} g_{x_{2}}^{\mathrm{q}_{2}} x_{2}^{\mathrm{k}} \cdots u_{m-1} x_{m}^{\mathrm{k}} g_{x_{m}}^{\mathrm{q}_{m}} x_{m}^{\mathrm{k}} u_{m}\right)\right) \\
& =\varphi\left(\hat{\mathrm{f}}\left(u_{0}^{\prime} x_{1}^{\mathrm{k}}\right) g_{x_{1}}^{\mathrm{q}_{1}} \hat{\mathrm{f}}\left(x_{1}^{\mathrm{k}} u_{1} x_{2}^{\mathrm{k}}\right) g_{x_{2}}^{\mathrm{q}_{2}} \cdots \hat{\mathrm{f}}\left(x_{m-1}^{\mathrm{k}} u_{m-1} x_{m}^{\mathrm{k}}\right) g_{x_{m}}^{\mathrm{q}_{m}} \hat{\mathrm{f}}\left(x_{m}^{\mathrm{k}} u_{m}\right)\right) .
\end{aligned}
$$

Analogously, $\phi(\rho)=\varphi\left(\grave{\mathrm{f}}\left(u_{m}^{\prime} x_{m+1}^{\mathrm{k}}\right) g_{x_{m+1}}^{q_{m+1}} \hat{\mathrm{f}}\left(x_{m+1}^{\mathrm{k}} u_{m+1} x_{m+2}^{\mathrm{k}}\right) g_{x_{m+2}}^{q_{m+2}} \cdots \hat{\mathrm{f}}\left(x_{n-1}^{\mathrm{k}} u_{n-1} x_{n}^{\mathrm{k}}\right) g_{x_{n}}^{q_{n}} \hat{\mathrm{f}}\left(x_{n}^{\mathrm{k}} u_{n}\right)\right)$. Let us now prove the following lemma.

Lemma 5.3 Let $i \in I_{m, n}, j \in\{1, m+1\}$ and $k \in\{m, n\}$.

(a) $\hat{\mathrm{f}}\left(x_{i}^{\mathrm{k}} u_{i} x_{i+1}^{\mathrm{k}}\right)=\mathrm{f}\left(x_{i}^{\mathrm{k}}\right) \mathrm{f}\left(x_{i}^{\mathrm{j}} u_{i} x_{i+1}^{\mathrm{j}}\right) \mathrm{f}\left(x_{i+1}^{\mathrm{k}}\right)^{t_{i}+1}$.

(b) If $u_{j-1}^{\prime}$ is the empty word, then $\grave{\mathrm{f}}\left(u_{j-1}^{\prime} x_{j}^{\mathrm{k}}\right)=x_{j}^{\mathrm{k}}$. Otherwise, $\grave{\mathrm{f}}\left(u_{j-1}^{\prime} x_{j}^{\mathrm{k}}\right)=u_{j-1}^{\prime} x_{j}^{r_{j}} x_{j}^{\prime} \mathrm{f}\left(x_{j}^{\mathrm{k}}\right)$ for some integer $₫ \leq r_{j}<\mathbb{k}$ and some proper prefix $x_{j}^{\prime}$ of $x_{j}$.

(c) If $u_{k}$ is the empty word, then $\dot{f}\left(x_{k}^{\mathrm{k}} u_{k}\right)=x_{k}^{\mathrm{k}}$. Otherwise, $\dot{\mathrm{f}}\left(x_{k}^{\mathrm{k}} u_{k}\right)=\mathrm{f}\left(x_{k}^{\mathrm{k}}\right) x_{k}^{\prime} x_{k}^{r_{k}} u_{k}$ for some integer $\stackrel{\therefore}{\leq} r_{k}<\mathbb{k}$ and some proper suffix $x_{k}^{\prime}$ of $x_{k}$. 
Proof. For $(a)$, write $w=x_{i}^{\mathrm{k}} u_{i} x_{i+1}^{\mathrm{k}}$ and notice that $w \notin L$, whence $\operatorname{sc}_{L}[w]$ is of the form $\operatorname{sc}_{L}[w]=\left(w_{0}, \ddot{w}_{1}, w_{1}, \ldots, \ddot{w}_{p}, w_{p}\right)$ with $p \geq 1$. Moreover, since $x_{i}^{\mathrm{k}} \dot{i}_{1}\left(u_{i} x_{i+1}\right), \mathrm{t}_{1}\left(x_{i} u_{i}\right) x_{i+1}^{\mathrm{k}} \notin L$, $w_{0}=x_{i}^{\mathrm{k}}$ and $w_{p}=x_{i+1}^{\mathrm{k}}$. By definition of $\mathrm{f}, \mathrm{f}\left(\ddot{w}_{j}\right)=\mathrm{f}\left(w_{j^{\prime}}\right)=1_{G}$ for every $j \in\{1, \ldots, p\}$ and $j^{\prime} \in\{1, \ldots, p-1\}$ such that $w_{j^{\prime}} \notin W_{j} \cup W_{\mathrm{k}}^{\prime}$. By definition of $\hat{\mathrm{f}}$, it results that

$$
\hat{\mathbf{f}}(w)=\mathbf{f}\left(w_{0}\right) \mathbf{f}\left(w_{j_{1}}\right) \cdots \mathbf{f}\left(w_{j_{r}}\right) \mathbf{f}\left(w_{p}\right),
$$

where $w_{0}, w_{j_{1}}, \ldots, w_{j_{r}}, w_{p}$ (with $0<j_{1}<\cdots<j_{r}<p$ ) is the sequence of all coordinates of $w$ that belong to the set $W_{\mathrm{j}} \cup W_{\mathrm{k}}^{\prime}$.

By definition of $t_{i}, x_{i}^{j} u_{i}=v_{i} x_{i+1}^{t_{i}}$ and $x_{i+1}$ is not a suffix of $v_{i}$. Hence $w=x_{i}^{\mathrm{k}-\mathrm{j}} v_{i} x_{i+1}^{\mathrm{k}+t_{i}}$ and $x_{i+1}^{\mathrm{k}}$ has $t_{i}+1$ maximal occurrences on the suffix $x_{i+1}^{\mathrm{k}+t_{i}}$ of $w$. It is then clear that $r \geq t_{i}$ and $w_{j_{r-t_{i}+1}}=\cdots=w_{j_{r}}=w_{p}=x_{i+1}^{\mathrm{k}}$. On the other hand, $x_{i}^{\mathrm{k}} u_{i} x_{i+1}^{\mathrm{k}}=x_{i}^{\mathrm{k}-\mathrm{j}} x_{i}^{\mathrm{j}} u_{i} x_{i+1}^{\mathrm{j}} x_{i+1}^{\mathrm{k}-\mathrm{j}}$ and $x_{i}^{j} u_{i} x_{i+1}^{j} \in W_{j}$. Moreover, since, by the choice of $\mathfrak{j}, x_{i}^{j} u_{i} x_{i+1}^{j}$ is synchronized in $x_{j}^{-\infty} u_{j} x_{j+1}^{+\infty}$ for every $j \in I_{m, n}$, the words $a_{i \ell_{i}} x_{i}^{j} u_{i} x_{i+1}^{j}$ and $x_{i}^{j} u_{i} x_{i+1}^{j} a_{i+11}$ do not belong to $L$. Hence, the word $x_{i}^{j} u_{i} x_{i+1}^{j} \in L$ has a maximal occurrence in $w$ and so $x_{i}^{j} u_{i} x_{i+1}^{j}=w_{j_{s}}$ for some $s$ such that $1 \leq s \leq r-t_{i}$. We claim that $1=s=r-t_{i}$ (whence $r=t_{i}+1$ ). This claim, together with (5.3), completes the proof of $(a)$. To show the claim, notice first that the above mentioned occurrence of $x_{i}^{j} u_{i} x_{i+1}^{j}$ is the unique occurrence of an element of $W_{j}$ in $w$ because these elements are synchronized in $x_{i}^{-\infty} u_{i} x_{i+1}^{+\infty}$. Suppose, on the other hand, that some element $x_{j}^{\mathrm{k}} \in W_{\mathrm{k}}^{\prime}$ occurs in $w$. Then, by the assumption on $\mathbb{k}, x_{j}^{\mathrm{k}}$ overlaps the prefix $x_{i}^{\mathrm{k}}$ or the suffix $x_{i+1}^{\mathrm{k}}$ of $w$ for a factor sufficiently large to deduce (from Fine and Wilf's Theorem and the fact that $x_{i}, x_{i+1}$ and $x_{j}$ are Lyndon words) that $x_{j}=x_{i}$ or $x_{j}=x_{i+1}$. Since, by definition of canonical form, $x_{i}$ is not a prefix of $u_{i} x_{i+1}^{j}$ and, as we have seen above, $x_{i+1}$ is not a suffix of $v_{i}$, this means that the occurrence of $x_{j}^{\mathrm{k}}$ determines one of the already identified coordinates $w_{0}, w_{j_{r-t_{i}+1}}, \ldots, w_{j_{r}}, w_{p}$ of $w$, thus proving that $j_{s}=j_{1}$ and $j_{r-t_{i}+1}=j_{2}$. This shows the claim and concludes the proof of $(a)$.

We now establish $(b)$, Let $w=u_{j-1}^{\prime} x_{j}^{\mathrm{k}}$. If $u_{j-1}^{\prime}$ is the empty word, then $w=x_{j}^{\mathrm{k}} \in L$, whence, by definition of the function $\grave{\mathrm{f}}, \mathfrak{\mathrm { f }}(w)=x_{j}^{\mathrm{k}}$. Suppose that $u_{j-1}^{\prime}$ is non-empty. Then $w \notin L$ and, so, $\operatorname{sc}_{L}[w]$ is of the form $\operatorname{sc}_{L}[w]=\left(w_{0}, \ddot{w}_{1}, w_{1}, \ldots, \ddot{w}_{p}, w_{p}\right)$ with $p \geq 1$. As $x_{j}^{\mathrm{k}}$ is the longest suffix of $w$ in $L, w_{p}=x_{j}^{\mathrm{k}}$. On the other hand, since $u_{j-1}^{\prime} x_{j}^{\mathrm{i}} \in W_{\mathrm{i}} \subseteq L$, the longest prefix of $w$ in $L$ is of the form $u_{j-1}^{\prime} x_{j}^{r_{j}} x_{j}^{\prime}$ for some integer $\stackrel{0}{i} r_{j}<\mathbb{k}$ and some proper prefix $x_{j}^{\prime}$ of $x_{j}$, whence $w_{0}=u_{j-1}^{\prime} x_{j}^{r_{j}} x_{j}^{\prime}$. By definition of canonical form and of the set $W_{j} \cup W_{\mathrm{k}}^{\prime}$ it is clear that the intermediate coordinates $\ddot{w}_{1}, w_{1}, \ldots, \ddot{w}_{p}$ of $w$ do not belong to this set. By definition of $\grave{\mathrm{f}}$ it then follows that $\grave{\mathrm{f}}(w)=w_{0} \mathrm{f}\left(w_{p}\right)=u_{j-1}^{\prime} x_{j}^{r_{j}} x_{j}^{\prime} \mathrm{f}\left(x_{j}^{\mathrm{k}}\right)$, thus proving $(b)$,

Condition (c) is symmetrical to condition $(b)$ and so it can be proved analogously, thus completing the proof of the lemma.

Lemma $5.3(a)$ and the definition of $\mathrm{f}$ show that, for every $i \in I_{m, n}$,

$$
\hat{\mathrm{f}}\left(x_{i}^{\mathrm{k}} u_{i} x_{i+1}^{\mathrm{k}}\right)=g_{x_{i}} g_{x_{i}}^{-1} g_{x_{i}, u_{i}, x_{i+1}} g_{x_{i+1}}^{-t_{i}-1} g_{x_{i+1}}^{t_{i}+1}=g_{x_{i}, u_{i}, x_{i+1}} .
$$

Combining this with conditions $(b)$ and $(c)$ of Lemma 5.3 and with the previous calculations, we may now finish the evaluation of $\pi$ and $\rho$ in $S_{\pi, \rho}$. Let $z_{0}^{\prime}$ be the first coordinate of $u_{0}^{\prime} x_{1}^{k}$ 
and $z_{m}$ be the last coordinate of $x_{m}^{\mathrm{k}} u_{m}$ determined by $L$. Let $h_{0}^{\prime}=g_{x_{1}}$ if $u_{0}^{\prime} \neq 1$ and $h_{0}^{\prime}=1_{G}$ otherwise. Analogously, let $h_{m}=g_{x_{m}}$ if $u_{m} \neq 1$ and $h_{m}=1_{G}$ otherwise. Then

$$
\begin{aligned}
\phi(\pi) & =\varphi\left(z_{0}^{\prime} h_{0}^{\prime} g_{x_{1}}^{q_{1}} g_{x_{1}, u_{1}, x_{2}} g_{x_{2}}^{\mathrm{q}_{2}} \cdots g_{x_{m-1}, u_{m-1}, x_{m}} g_{x_{m}}^{\mathrm{q}_{m}} h_{m} z_{m}\right) \\
& =\left(z_{0}^{\prime}, h_{0}^{\prime} g_{x_{1}}^{\mathrm{q}_{1}} g_{x_{1}, u_{1}, x_{2}} g_{x_{2}}^{\mathrm{q}_{2}} \cdots g_{x_{m-1}, u_{m-1}, x_{m}} g_{x_{m}}^{\mathrm{q}_{m}} h_{m}, z_{m}\right) \\
& =\left(z_{0}^{\prime}, g_{\pi}, z_{m}\right)
\end{aligned}
$$

and, analogously, $\phi(\rho)=\left(z_{m}^{\prime}, g_{\rho}, z_{n}\right)$. Since $S_{\pi, \rho}$ verifies the $\kappa$-identity $\pi=\rho$, by hypothesis, we have that $\phi(\pi)=\phi(\rho)$. Then $z_{0}^{\prime}=z_{m}^{\prime}, g_{\pi}=g_{\rho}$ and $z_{m}=z_{n}$. From $g_{\pi}=g_{\rho}$ we deduce that the identity $\mathrm{w}_{\pi}=\mathrm{w}_{\rho}$ is trivial. In particular, $\mathrm{u}_{0}^{\prime} \mathrm{v}_{x_{1}}^{\mathrm{q}_{1}}=\mathrm{u}_{m}^{\prime} \mathrm{v}_{x_{m+1}}^{\mathrm{q}_{m+1}}$ and, so, $x_{1}=x_{m+1}$. Now, it results from the equality $z_{0}^{\prime}=z_{m}^{\prime}$ and the fact that $\pi$ and $\rho$ are in canonical form that $u_{0}^{\prime}=u_{m}^{\prime}$. By symmetry, we also have $u_{m}=u_{n}$. This completes the proof that $(c)$ implies $(d)$.

The following decidability result may now be easily deduced.

Corollary 5.4 Given $\kappa$-terms $\pi$ and $\rho$ of rank at most 1, it is decidable whether LG (resp. S) satisfies $\pi=\rho$.

Proof. Since LG contains the pseudovariety $\mathbf{L I}$ and $\mathbf{L I}$ separates different finite words as well as finite words from rank $1 \kappa$-terms, if one of $\pi$ and $\rho$ is a finite word then they both are the same finite word. So, we assume that $\pi$ and $\rho$ are both rank $1 \kappa$-terms. In view of Theorem 5.1, to decide whether $\mathbf{L G}$ (resp. $\mathbf{S}$ ) verifies $\pi=\rho$ it suffices to compute the canonical forms $\pi^{\prime}$ and $\rho^{\prime}$ of $\pi$ and $\rho$, respectively, and to verify if $\pi^{\prime}$ and $\rho^{\prime}$ are the same $\kappa$-term.

\section{An alternative version of Theorem 5.1}

By way of comparison, we present an alternative version of Theorem 5.1. This second version contains some adjustments in the construction of the group identity $\mathrm{w}_{\pi}=\mathrm{w}_{\rho}$ and of the testsemigroup $S_{\pi, \rho}$ associated with a given $\kappa$-identity $\pi=\rho$. Although in [7] we will follow the first scheme to complete the proof of the decidability of the word problem for $\kappa$-terms over $\mathbf{L G}$, this new construction is less tricky and has some advantages that may be exploited in future work. Here, the test-semigroup $S_{\pi, \rho}$ is of the form $S_{k}(G, \mathrm{f})$ and the group identity $\mathrm{w}_{\pi}=\mathrm{w}_{\rho}$ involves -1 exponents and is not reduced in the free group. Instead of $S_{k}(G, \mathrm{f})$, we could use a semigroup $M_{k}(G, \hbar)$ with exactly the same effect. Actually, the group identity $\mathrm{w}_{\pi}=\mathrm{w}_{\rho}$ is the one that would emerge if one would use the representation of the free pro- $\left(\mathbf{G} * \mathbf{D}_{k}\right)$ semigroups obtained by Almeida and Azevedo [2].

We let $\pi=u_{0}^{\prime} x_{1}^{\omega+q_{1}} u_{1} \cdots x_{m}^{\omega+q_{m}} u_{m}$ and $\rho=u_{m}^{\prime} x_{m+1}^{\omega+q_{m+1}} u_{m+1} \cdots x_{n}^{\omega+q_{n}} u_{n}$ be rank $1 \kappa$-terms in canonical form and assume the same notations $I_{n}=\{1, \ldots, n\}, I_{m, n}=I_{n} \backslash\{m, n\}, \ell_{i}=\left|x_{i}\right|$, $x_{i}=a_{i 1} a_{i 2} \cdots a_{i \ell_{i}}$ and $x_{i j}=a_{i j} \cdots a_{i \ell_{i}} a_{i 1} \cdots a_{i j-1}$ of the previous section. Now, let $\mathbb{k} \in \mathbb{N}$ be such that $\mathbb{k}+1=r \ell_{1} \ell_{2} \cdots \ell_{n}$, with $r>1+\max \left\{\left|u_{0}^{\prime}\right|,\left|u_{m}^{\prime}\right|,\left|u_{j}\right|,\left|q_{j}\right|: j \in I_{n}\right\}$, and let 
$r_{i}=r \ell_{1} \cdots \ell_{i-1} \ell_{i+1} \cdots \ell_{n}=\frac{\mathrm{k}+1}{\ell_{i}}$. Denote at last $b_{i j}=x_{i j}^{r_{i}}, b_{i 1}^{\prime}=i_{\mathrm{k}}\left(b_{i 1}\right)=x_{i}^{r_{i}-1} a_{i 1} a_{i 2} \cdots a_{i \ell_{i}-1}$ and $b_{i 1}^{\prime \prime}=\mathrm{t}_{\mathrm{k}}\left(b_{i 1}\right)=a_{i 2} a_{i 3} \cdots a_{i \ell_{i}} x_{i}^{r_{i}-1}$.

We define first the group identity $\mathrm{w}_{\pi}=\mathrm{w}_{\rho}$. Let $L_{\mathrm{k}}=A^{\leq \mathrm{k}}$, whence $\ddot{L}_{\mathrm{k}}=A^{\mathrm{k}+1}$. We associate to each word $u \in \ddot{L}_{\mathrm{k}}$ a variable $\mathrm{v}_{u}$, let $\mathrm{V}=\left\{\mathrm{v}_{u} \mid u \in \ddot{L}_{\mathrm{k}}\right\}$ and denote by $F_{\mathrm{V}}$ the free group generated by the alphabet $\vee$. Let $\lambda: A^{+} \rightarrow F_{\bigvee}$ be the $\mathbb{k}_{\text {-superposition homomorphism }}$ given by $\lambda(u)=\mathrm{v}_{u}$ for every $u \in \ddot{L}_{\mathrm{k}}$, whence for a word $v \in A^{+} \backslash L_{\mathrm{k}}, \lambda(v)=\mathrm{v}_{v_{1}} \mathrm{v}_{v_{2}} \cdots \mathrm{v}_{v_{|v|-k}}$ where $v_{1}, v_{2}, \ldots, v_{|v|-\mathrm{k}} \in \ddot{L}_{\mathrm{k}}$ are the successive factors of length $\mathbb{k}+1$ of $v$. Now, let

$$
\mathrm{w}_{\pi}=\mathrm{w}_{0}^{\prime} \mathrm{y}_{1}^{q_{1}-r_{1}} \mathrm{w}_{1} \cdots \mathrm{y}_{m}^{q_{m}-r_{m}} \mathrm{w}_{m} \quad \text { and } \quad \mathrm{w}_{\rho}=\mathrm{w}_{m}^{\prime} \mathrm{y}_{m+1}^{q_{m+1}-r_{m+1}} \mathrm{w}_{m+1} \cdots \mathrm{y}_{n}^{q_{n}-r_{n}} \mathrm{w}_{n}
$$

where, for $i \in I_{n}$ and $j \in\{0, m\}, \mathrm{y}_{i}=\lambda\left(x_{i 1} b_{i 1}^{\prime}\right), \mathrm{w}_{j}^{\prime}=\lambda\left(u_{j}^{\prime} b_{j+1 j+1}^{\prime}\right), \mathrm{w}_{i}=\lambda\left(b_{i 1} u_{i}\right)$ if $i \in\{m, n\}$ and $\mathrm{w}_{i}=\lambda\left(b_{i 1} u_{i} b_{i+11}^{\prime}\right)$ otherwise. We denote by $\widetilde{\mathrm{w}}_{\pi}$ and $\widetilde{\mathrm{w}}_{\rho}$, respectively, the reduced forms of $\mathrm{w}_{\pi}$ and $\mathrm{w}_{\rho}$ in $F_{\mathrm{V}}$.

Let us now define the test-semigroup $S_{\pi, \rho}$. Choose a group homomorphism $\eta: F_{\vee} \rightarrow G$ into a finite group $G$ such that $\eta\left(\mathrm{w}_{\pi}\right) \neq \eta\left(\mathrm{w}_{\rho}\right)$ when $\widetilde{\mathrm{w}}_{\pi} \neq \widetilde{\mathrm{w}}_{\rho}$. Then let $\mathrm{f}: L_{\mathrm{k}} \cup \ddot{L}_{\mathrm{k}} \rightarrow G$ be the mapping defined by $\mathrm{f}\left(L_{\mathrm{k}}\right)=\left\{1_{G}\right\}$ and $\mathrm{f}(u)=\eta\left(\mathrm{v}_{u}\right)=\eta(\lambda(u))$ for each $u \in \ddot{L}_{\mathrm{k}}$. Finally, we let $S_{\pi, \rho}=S_{\mathrm{k}}(G, \mathrm{f})$. Recall that condition $\mathrm{f}\left(L_{\mathrm{k}}\right)=\left\{1_{G}\right\}$ makes the function $\hat{\mathrm{f}}$ define a k-superposition homomorphism from $A^{+}$into $G$ (the $\mathbb{k}$-superposition property is a particular case of the property presented in Lemma $2.3(a)$.

The announced alternative version of Theorem 5.1 is the following.

Theorem 6.1 Under the above assumptions and with the above notations, the following conditions are equivalent:

(a) $\mathbf{S} \models \pi=\rho$.

(b) $\mathbf{L G} \models \pi=\rho$.

(c) $S_{\pi, \rho} \models \pi=\rho$.

(d) $\widetilde{\mathrm{w}}_{\pi}=\widetilde{\mathrm{w}}_{\rho}, u_{0}^{\prime}=u_{m}^{\prime}$ and $u_{m}=u_{n}$.

(e) $\pi$ and $\rho$ are the same $\kappa$-term.

Proof. The implications $(e) \Rightarrow(a) \Rightarrow(b) \Rightarrow(c)$ hold trivially. To prove the implication $(c) \Rightarrow(d)$, assume that $S_{\pi, \rho} \models \pi=\rho$ and let $\phi_{\mathrm{k}}: A^{+} \rightarrow S_{\mathrm{k}}(G, \mathrm{f})$ be the homomorphism $\phi_{\mathrm{k}}=\varphi \circ \check{\mathrm{f}}$, where $\varphi$ is the isomorphism from $\mathcal{Z}\left[G, L_{\mathrm{k}}, \mathrm{f}\right]$ onto $S_{\pi, \rho}$ defined in Section 2, and notice that $\phi_{\mathrm{k}}(w)=\left(\mathbf{i}_{\mathrm{k}}(w), \hat{\mathrm{f}}(w), \mathrm{t}_{\mathrm{k}}(w)\right)$ for $w \in A^{+} \backslash L_{\mathrm{k}}$. We use the same notation for the homomorphism of $\kappa$-semigroups $T_{A}^{\kappa} \rightarrow S_{\pi, \rho}$ that coincides with $\phi_{\mathrm{k}}$ on $A^{+}$.

Since $S_{\pi, \rho}$ is a finite semigroup, it verifies the identity $x^{p !}=x^{\omega}$ for every sufficiently large positive integer $p$. Take one such $p$ with $p !+q_{i} \geq \mathbb{k}+1$ for all $i \in I_{n}$. Then, $\phi_{\mathrm{k}}(\pi)=\phi_{\mathrm{k}}\left(\pi_{p}\right)$ and $\phi_{\mathrm{k}}(\rho)=\phi_{\mathrm{k}}\left(\rho_{p}\right)$ where $\pi_{p}$ and $\rho_{p}$ are the words from $A^{+}$obtained, respectively, from $\pi$ and $\rho$ by replacing each occurrence of $\omega$ by $p$ !. Since $\hat{f}$ is a $\mathbb{k}$-superposition homomorphism, $\phi_{\mathrm{k}}\left(x_{i}^{p !+q_{i}}\right)=\left(b_{i 1}^{\prime},\left(\mathrm{f}\left(b_{i 1}\right) \mathrm{f}\left(b_{i 2}\right) \cdots \mathrm{f}\left(b_{i \ell_{i}}\right)\right)^{p !+q_{i}-r_{i}} \mathrm{f}\left(b_{i 1}\right), b_{i 1}^{\prime \prime}\right)$. On the other hand 
$\left(\mathrm{f}\left(b_{i 1}\right) \mathrm{f}\left(b_{i 2}\right) \cdots \mathrm{f}\left(b_{i \ell_{i}}\right)\right)^{p !+q_{i}-r_{i}}=\left(\mathrm{f}\left(b_{i 1}\right) \mathrm{f}\left(b_{i 2}\right) \cdots \mathrm{f}\left(b_{i \ell_{i}}\right)\right)^{q_{i}-r_{i}}$ because $G$ is a subgroup of $S_{\pi, \rho}$ and, so, it verifies the identity $x^{p !}=1$. Using again the fact that $\hat{f}$ is a $\mathbb{k}$-superposition homomorphism, it follows that

$$
\phi_{\mathrm{k}}(\pi)=\phi_{\mathrm{k}}\left(\pi_{p}\right)=\left(\mathrm{i}_{\mathrm{k}}\left(u_{0}^{\prime} b_{11}\right), w_{0}^{\prime} y_{1}^{q_{1}-r_{1}} w_{1} \cdots y_{m}^{q_{m}-r_{m}} w_{m}, \mathrm{t}_{\mathrm{k}}\left(b_{m 1} u_{m}\right)\right)
$$

where $y_{i}=\mathbf{f}\left(b_{i 1}\right) \mathbf{f}\left(b_{i 2}\right) \cdots \mathbf{f}\left(b_{i \ell_{i}}\right)=\hat{\mathbf{f}}\left(x_{i 1} b_{i 1}^{\prime}\right), w_{0}^{\prime}=\hat{\mathbf{f}}\left(u_{0}^{\prime} b_{11}^{\prime}\right), w_{m}=\mathbf{f}\left(b_{m 1}\right) \hat{\mathbf{f}}\left(b_{m 1}^{\prime \prime} u_{m}\right)=\hat{\mathbf{f}}\left(b_{m 1} u_{m}\right)$ and, for $i \neq m, w_{i}=\mathrm{f}\left(b_{i 1}\right) \hat{\mathrm{f}}\left(b_{i 1}^{\prime \prime} u_{i} b_{i+11}^{\prime}\right)=\hat{\mathrm{f}}\left(b_{i 1} u_{i} b_{i+11}^{\prime}\right)$. Analogously,

$$
\phi_{\mathrm{k}}(\rho)=\phi_{\mathrm{k}}\left(\rho_{p}\right)=\left(\mathrm{i}_{\mathrm{k}}\left(u_{m}^{\prime} b_{m+11}\right), w_{m}^{\prime} y_{m+1}^{q_{m+1}-r_{m+1}} w_{m+1} \cdots y_{n}^{q_{n}-r_{n}} w_{n}, \mathrm{t}_{\mathrm{k}}\left(b_{n 1} u_{n}\right)\right) .
$$

As one may confirm easily, the following equalities hold

$$
\begin{aligned}
& \eta\left(\mathrm{w}_{\pi}\right)=w_{0}^{\prime} y_{1}^{q_{1}-r_{1}} w_{1} \cdots y_{m}^{q_{m}-r_{m}} w_{m}=\hat{\mathrm{f}}\left(\pi_{p}\right), \\
& \eta\left(\mathrm{w}_{\rho}\right)=w_{m}^{\prime} y_{m+1}^{q_{m+1}-r_{m+1}} w_{m+1} \cdots y_{n}^{q_{n}-r_{n}} w_{n}=\hat{\mathrm{f}}\left(\rho_{p}\right) .
\end{aligned}
$$

By hypothesis $S_{\pi, \rho}$ verifies $\pi=\rho$, whence $\phi_{\mathrm{k}}(\pi)=\phi_{\mathrm{k}}(\rho)$. Thus $i_{\mathrm{k}}\left(u_{0}^{\prime} b_{11}\right)=i_{\mathrm{k}}\left(u_{m}^{\prime} b_{m+11}\right)$, $\eta\left(\mathrm{w}_{\pi}\right)=\eta\left(\mathrm{w}_{\rho}\right)$ and $\mathrm{t}_{\mathrm{k}}\left(b_{m 1} u_{m}\right)=\mathrm{t}_{\mathrm{k}}\left(b_{n 1} u_{n}\right)$. The second condition $\eta\left(\mathrm{w}_{\pi}\right)=\eta\left(\mathrm{w}_{\rho}\right)$ and the definition of $\eta$ imply that $\mathbf{G} \models \mathrm{w}_{\pi}=\mathrm{w}_{\rho}$ and, so, that the identity $\widetilde{\mathrm{w}}_{\pi}=\widetilde{\mathrm{w}}_{\rho}$ is trivial. In particular $\mathrm{w}_{0}^{\prime} \mathrm{y}_{1}^{q_{1}-r_{1}}=\mathrm{w}_{m}^{\prime} \mathrm{y}_{m+1}^{q_{m+1}-r_{m+1}}$. Since $r$ may be chosen arbitrarily large the same happens with every $r_{i}$. Hence, each exponent $q_{i}-r_{i}$ is negative, with absolute value as large as we want. Therefore, as $\pi$ and $\rho$ are in canonical form, one deduces easily that $\mathrm{w}_{0}^{\prime}=\mathrm{w}_{m}^{\prime}$ and $\mathrm{y}_{1}=\mathrm{y}_{m+1}$ and then that $x_{1}=x_{m+1}$. Now, the first condition $\mathrm{i}_{\mathrm{k}}\left(u_{0}^{\prime} b_{11}\right)=\mathrm{i}_{\mathrm{k}}\left(u_{m}^{\prime} b_{m+11}\right)$ above immediately implies that $u_{0}^{\prime}=u_{m}^{\prime}$. Symmetrically, one deduces that $u_{m}=u_{n}$. This shows the implication $(c) \Rightarrow(d)$.

Let us now prove that $(d)$ implies $(e)$. Assume that $\widetilde{\mathrm{w}}_{\pi}=\widetilde{\mathrm{w}}_{\rho}$ is a trivial identity and that $u_{0}^{\prime}=u_{m}^{\prime}$ and $u_{m}=u_{n}$. For $j \in I_{m, n}$ let $u_{j}=x_{j}^{\prime} v_{j}^{\prime \prime}=v_{j}^{\prime} x_{j+1}^{\prime \prime}$ be two factorizations of $u_{j}$ where $x_{j}^{\prime}$ (resp. $x_{j+1}^{\prime \prime}$ ) is the longest common prefix (resp. suffix) of $u_{j}$ and $x_{j}$ (resp. $x_{j+1}$ ). Consider the words $z_{j}^{\prime \prime}=\mathrm{t}_{\mathrm{k}-\left|x_{j}^{\prime}\right|}\left(x_{j}^{\mathrm{k}}\right)$ and $z_{j+1}^{\prime}=i_{\mathrm{k}-\left|x_{j+1}^{\prime \prime}\right|}\left(x_{j+1}^{\mathrm{k}}\right)$. With this notation and since $\lambda$ is a $\mathbb{k}$-superposition homomorphism, we have

$$
\begin{aligned}
\mathrm{w}_{j} & =\lambda\left(b_{j 1} x_{j}^{\prime} v_{j}^{\prime \prime} b_{j+11}^{\prime}\right)=\lambda\left(b_{j 1} x_{j}^{\prime}\right) \lambda\left(\mathrm{t}_{\mathrm{k}}\left(b_{j 1} x_{j}^{\prime}\right) v_{j}^{\prime \prime} b_{j+11}^{\prime}\right) \\
& =\lambda\left(b_{j 1} x_{j}^{\prime}\right) \lambda\left(z_{j}^{\prime \prime} x_{j}^{\prime} v_{j}^{\prime \prime} b_{j+11}^{\prime}\right)=\lambda\left(b_{j 1} x_{j}^{\prime}\right) \lambda\left(z_{j}^{\prime \prime} v_{j}^{\prime} x_{j+1}^{\prime \prime} b_{j+11}^{\prime}\right) \\
& =\lambda\left(b_{j 1} x_{j}^{\prime}\right) \lambda\left(z_{j}^{\prime \prime} u_{j} z_{j+1}^{\prime}\right) \lambda\left(x_{j+1}^{\prime \prime} b_{j+11}^{\prime}\right) .
\end{aligned}
$$

By the choice of $x_{j}^{\prime}, \lambda\left(b_{j 1} x_{j}^{\prime}\right)$ is the longest prefix of $\mathrm{w}_{j}$ that is canceled by $\mathrm{y}_{j}^{q_{j}-r_{j}}$ in the reduction process of $\mathrm{w}_{\pi}$ (if $j<m$ ) and $\mathrm{w}_{\rho}$ (otherwise). Dually, $\lambda\left(x_{j+1}^{\prime \prime} b_{j+11}^{\prime}\right)$ is the longest suffix of $\mathrm{w}_{j}$ that is canceled by $\mathrm{y}_{j+1}^{q_{j+1}-r_{j+1}}$. Moreover, $\lambda\left(b_{j 1} x_{j}^{\prime}\right)$ and $\lambda\left(x_{j+1}^{\prime \prime} b_{j+11}^{\prime}\right)$ are words over the alphabet $\mathrm{V}$ of length, respectively, $\left|x_{j}^{\prime}\right|+1$ and $\left|x_{j+1}^{\prime \prime}\right|$ and so less than or equal to $\left|u_{j}\right|$. Since each exponent $q_{i}-r_{i}$ is negative and with a large absolute value, we may assume that some (large) factor $\mathrm{y}_{j}^{\prime}$ of $\mathrm{y}_{j}^{q_{j}-r_{j}}$ remains in the reduced form. On the other hand, $\lambda\left(z_{j}^{\prime \prime} u_{j} z_{j+1}^{\prime}\right) \neq 1$ since $\left|z_{j}^{\prime \prime} u_{j} z_{j+1}^{\prime}\right|>\mathbb{k}$. Whence, the reduced forms $\widetilde{\mathrm{w}}_{\pi}$ and $\widetilde{\mathrm{w}}_{\rho}$ have precisely, respectively, $m-1$ and $n-m-1$ factors of $\mathrm{V}^{+}$that are placed between factors of $\left(\mathrm{V}^{-1}\right)^{+}$. 
Since $\widetilde{\mathrm{w}}_{\pi}=\widetilde{\mathrm{w}}_{\rho}$ by hypothesis, it follows that $m=n-m$ and, for every $j \in\{1, \ldots, m-1\}$, $\lambda\left(z_{j}^{\prime \prime} u_{j} z_{j+1}^{\prime}\right)=\lambda\left(z_{m+j}^{\prime \prime} u_{m+j} z_{m+j+1}^{\prime}\right)$, whence $z_{j}^{\prime \prime} u_{j} z_{j+1}^{\prime}=z_{m+j}^{\prime \prime} u_{m+j} z_{m+j+1}^{\prime}$. As $\mathbb{k}$ (and, so, also the words $z_{j}^{\prime \prime}, z_{j+1}^{\prime}, z_{m+j}^{\prime \prime}$ and $\left.z_{m+j+1}^{\prime}\right)$ is arbitrarily large, one deduces that the bi-infinite words $x_{j}^{-\infty} u_{j} x_{j+1}^{+\infty}$ and $x_{m+j}^{-\infty} u_{m+j} x_{m+j+1}^{+\infty}$ coincide. Hence, as a consequence of the hypothesis $u_{m}=u_{n}$ and of the rank 1 canonical form definition, for every $j \in\{1, \ldots, m\}, x_{j}=x_{m+j}$ and $u_{j}=u_{m+j}$, and thus $w_{j}=w_{m+j}, y_{j}=y_{m+j}$ and $r_{j}=r_{m+j}$. To deduce that $\pi$ and $\rho$ coincide, and since by hypothesis $u_{0}^{\prime}=u_{m}^{\prime}$, it remains to prove that $q_{j}=q_{m+j}$ for every $j \in\{1, \ldots, m\}$. But that is immediate after the above equalities and the fact that $\widetilde{\mathrm{w}}_{\pi}=\widetilde{\mathrm{w}}_{\rho}$. This shows the implication $(d) \Rightarrow(e)$ and concludes the proof of Theorem 6.1

Final remarks. The semigroups $\mathcal{S}(G, L, \mathrm{f})$ were used above as test-semigroups to show that the word problem for $\kappa$-terms of rank at most 1 over $\mathbf{L G}$ is decidable, and they are used in [7] to extend this result to any rank. Exploring the fact that $\mathbf{L H}$ is generated by the semigroups $\mathcal{S}(G, L, \mathrm{f})$ with $G \in \mathbf{H}$ and $L$ finite, we expect that the above method can be adapted to solve the $\kappa$-word problem over $\mathbf{L H}$, for other pseudovarieties $\mathbf{H}$ of groups.

\section{Acknowledgments}

This work was supported by the European Regional Development Fund, through the programme COMPETE, and by the Portuguese Government through FCT - Fundação para a Ciência e a Tecnologia, under the project PEst-C/MAT/UI0013/2011.

\section{References}

[1] J. Almeida, Finite semigroups and universal algebra, World Scientific, Singapore, 1995.

[2] J. Almeida and A. Azevedo, On regular implicit operations, Portugaliæ Mathematica 50 (1993), 35-61.

[3] J. Almeida, J. C. Costa and M. Zeitoun, McCammond's normal forms for free aperiodic semigroups revisited, Tech. Report CMUP 2012-3, Univ. Porto, 2012, Submitted.

[4] J. C. Costa, Free profinite locally idempotent and locally commutative semigroups, Journal of Pure and Applied Algebra 163 (2001), 19-47.

[5] J. C. Costa, Canonical forms for free א-semigroups, Discrete Math. Theor. Comput. Sci., To appear.

[6] J. C. Costa and C. Nogueira, Complete reducibility of the pseudovariety LSl, Int. J. Algebra and Computation 19 (2009), 247-282.

[7] J. C. Costa, C. Nogueira and M. L. Teixeira, The word problem for $\kappa$-terms over the pseudovariety of local groups, In preparation. 
[8] J. C. Costa and M. L. Teixeira, Tameness of the pseudovariety LSl, Int. J. Algebra and Computation 14 (2004), 627-654.

[9] J. Howie and N. Ruškuc, Constructions and presentations for monoids, Comm. Algebra 22 (1994), 6209-6224.

[10] G. Lallement, Semigroups and Combinatorial Applications, John Wiley, New York, 1979.

[11] M. Lothaire, Algebraic combinatorics on words, Cambridge University Press, Cambridge, UK, 2002.

[12] J. McCammond, Normal forms for free aperiodic semigroups, Int. J. Algebra Comput. 11 (2001), 581-625.

[13] H. Straubing, Finite semigroup varieties of the form $\mathbf{V} * \mathbf{D}$, J. Pure Appl. Algebra 36 (1985), 53-94. 